\title{
Lumiracoxib: the evidence of its clinical impact on the treatment of osteoarthritis
}

\author{
Louise Profit, Paul Chrisp \\ Core Medical Publishing, Knutsford, UK
}

\begin{abstract}
Introduction: The symptoms of osteoarthritis $(\mathrm{OA})$ include joint pain, stiffness, and a reduced ability to perform normal daily activities, which result in decreased quality of life. There is currently no known cure or means of preventing the progression of joint damage due to OA. Therefore, treatment focuses on the control of symptoms, including the use of various agents [including nonselective and selective nonsteroidal antiinflammatory drugs (NSAIDs)] to provide pain relief and reduce inflammation. Lumiracoxib is a selective cyclooxygenase-2 (COX-2) inhibitor for the treatment of OA.
\end{abstract}

Aims: To review the evidence for the treatment of OA with lumiracoxib.

Evidence review: There is evidence that lumiracoxib reduces the pain and stiffness associated with $O A$, and is as effective as nonselective NSAIDs, and the COX-2 inhibitor celecoxib. There is some evidence that lumiracoxib treatment results in a lower incidence of upper gastrointestinal (GI) ulcer complications compared with nonselective NSAIDs. However, evidence suggests that there is no GI benefit in patients receiving concomitant aspirin medication. With the exception of Gl ulcers, the evidence indicates that lumiracoxib has a tolerability profile similar to nonselective NSAIDs: low risk of cardiovascular (CV) events and a low incidence of edema. Changes in liver function occur in some patients, largely at doses $>100 \mathrm{mg}$. The cost effectiveness of lumiracoxib compared with nonselective NSAIDs remains to be determined.

Clinical value: Lumiracoxib is an alternative treatment option for OA which provides effective pain relief without the Gl complications associated with nonselective NSAIDs, and with a low risk of CV events. Lumiracoxib is contraindicated in patients with current, previous, or at risk of, hepatic impairment.

Core Evidence. 2007;2(2):131-150.

Key words: cardiovascular, COX-2 inhibitor, evidence, gastrointestinal, lumiracoxib, osteoarthritis, outcomes, treatment

\section{Core evidence clinical impact summary for lumiracoxib in osteoarthritis}

\begin{tabular}{|c|c|c|}
\hline Outcome measure & Evidence & Implications \\
\hline \multicolumn{3}{|l|}{ Patient-oriented evidence } \\
\hline Reduced pain intensity & Clear & $\begin{array}{l}\text { Effective relief of pain intensity, comparable with celecoxib, rofecoxib, diclofenac, } \\
\text { naproxen, and ibuprofen }\end{array}$ \\
\hline Improvement in joint stiffness & Clear & $\begin{array}{l}\text { Improvement in joint stiffness (WOMAC score) comparable with celecoxib and } \\
\text { diclofenac }\end{array}$ \\
\hline Improved quality of life & Clear & $\begin{array}{l}\text { Improvement in ability to perform daily activities (WOMAC score) comparable } \\
\text { with celecoxib and diclofenac. Improvement in physical functioning and mental } \\
\text { health (SF-36) compared with placebo }\end{array}$ \\
\hline Tolerability & Clear & $\begin{array}{l}\text { Minor Gl adverse events are frequent (e.g. dyspepsia, diarrhea, and nausea) and } \\
\text { occur at a similar incidence with celecoxib, diclofenac, ibuprofen, and naproxen }\end{array}$ \\
\hline Low incidence of Gl ulcers & Substantial & $\begin{array}{l}\text { Lower risk of upper GI ulcer complications compared with ibuprofen or naproxen } \\
\text { in nonaspirin population only }\end{array}$ \\
\hline Changes in liver function & Substantial & $\begin{array}{l}\text { No clinically relevant elevations in ALT/AST levels were reported at the indicated } \\
\text { dose of lumiracoxib ( } 100 \mathrm{mg} \text { ) although isolated cases of liver-related adverse events } \\
\text { have been reported at this dose. AST/ALT elevations may occur at higher doses } \\
\text { ( } 400 \mathrm{mg} \text { ). Lumiracoxib should not be used in patients with, or at risk of, liver } \\
\text { disease, and liver function should be monitored. }\end{array}$ \\
\hline Reduced risk of hypertension & Limited & $\begin{array}{l}\text { Lower risk of de-novo hypertension with lumiracoxib than naproxen. Reduced SBP } \\
\text { and DBP with lumiracoxib compared with naproxen/ibuprofen }\end{array}$ \\
\hline Low risk of CV events & $\begin{array}{l}\text { Moderate (limited in } \\
\text { patients at high risk) }\end{array}$ & $\begin{array}{l}\text { Lumiracoxib has a similar CV risk profile as ibuprofen, and a similar CV profile } \\
\text { as naproxen in patients taking aspirin. Lumiracoxib has an increased risk of CV } \\
\text { events compared with naproxen in patients not taking aspirin }\end{array}$ \\
\hline
\end{tabular}




\begin{tabular}{|lll|}
\hline $\begin{array}{l}\text {...table continued } \\
\text { Outcome measure }\end{array}$ & Evidence & Implications \\
\hline $\begin{array}{l}\text { Low incidence of edema } \\
\begin{array}{l}\text { Economic evidence } \\
\text { Cost effectiveness }\end{array}\end{array}$ & Substantial & Incidence similar to that with celecoxib, diclofenac, naproxen, and ibuprofen \\
& No evidence & $\begin{array}{l}\text { Evidence required to determine whether clinical effectiveness translates into } \\
\text { an economic benefit compared with other treatments for OA }\end{array}$ \\
\hline $\begin{array}{l}\text { ALT, alanine aminotransferase; AST, aspartate aminotransferase; CV, cardiovascular; DBP, diastolic blood pressure; GI, gastrointestinal; OA, osteoarthritis; SBP, systolic blood pressure; } \\
\text { SF, Short Form; WOMAC, Western Ontario and McMaster Universities OA Index. }\end{array}$ & \\
\hline
\end{tabular}

\section{Scope, aims, and objectives}

Osteoarthritis $(O A)$ is one of the most prevalent forms of arthritis. There is currently no known cure or means of preventing OA. Therefore, treatment focuses on the control of symptoms, including the use of the nonselective nonsteroidal antiinflammatory drugs (NSAIDs) and selective cyclooxygenase-2 (COX-2) inhibitors. The COX-2 inhibitors were developed with the aim of providing pain relief with less gastrointestinal (Gl) adverse effects than the NSAIDs. In recent years, there have been concerns regarding the cardiovascular (CV) safety of COX-2 inhibitors, and there is a need for newer agents that demonstrate a clinical benefit in a greater proportion of OA patients compared with the risk of a CV event. Lumiracoxib (Prexige ${ }^{\circledR}$, COX-189; Novartis) is an orally active selective COX-2 inhibitor developed for the treatment of $O A$, rheumatoid arthritis (RA), and acute pain.

The objective of this article is to review the evidence base for the clinical use and CV risk-benefit profile of lumiracoxib in the management of patients with $\mathrm{OA}$. The use of lumiracoxib in acute pain is excluded.

\section{Methods}

English language medical literature databases were searched for relevant evidence related to the treatment of OA with lumiracoxib. The searches were conducted in December 2005 using the search terms "lumiracoxib OR Prexige." The cut-off date was from the beginning of the database to the date of the search unless otherwise stated.

- PubMed, http://www.ncbi.nlm.nih.gov/entrez/query.fgci

- EMBASE, http://datastarweb.com

- BIOSIS, http://datastarweb.com

- Database of Abstracts of Reviews of Effects (DARE), http://www.york.ac.uk/inst/crd/darehp.htm

- Cochrane Database of Systematic Reviews (CDSR), http://www.cochrane.org/index0.htm

- Public Library of Science, http://clinicaltrials.plosjournals.org/

- Clinical Evidence (BMJ), http://www.clinicalevidence.com

- http://www.clinicaltrials.gov

- http://www.clinicalstudyresults.org. Search strategy "Novartis AND lumiracoxib"
- National Institute for Health and Clinical Excellence (NICE), http://www.nice.org.uk

- National Guideline Clearinghouse, http://www.guideline.gov. Search strategy "osteoarthritis"

After removal of duplicates, a total of 235 records were identified. Clinical guidelines for OA were also identified from NICE, the American College of Rheumatology (ACR), and the European League Against Rheumatism (EULAR). Records were manually reviewed and any animal studies, in-vitro studies, news articles, nonsystematic reviews, editorials, articles that mentioned lumiracoxib but did not discuss clinical trial data, duplicated data presented in previous publications, pharmacokinetic and interaction studies which were not directly relevant to the review, and studies with lumiracoxib in indications other than OA were excluded. Eighteen papers remained and were included in the evidence base (Table 1). Two systematic reviews were identified. All other articles were of level 2 evidence reported as full publications.

\section{Table 1 | Evidence base included in the review}

\begin{tabular}{|lcc|}
\hline Category & \multicolumn{2}{c|}{ Number of records } \\
\cline { 2 - 3 } & Full papers & Abstracts \\
\hline Initial search & 235 & 32 \\
records excluded & 217 & 28 \\
records included & 18 & 4 \\
Search updates, new records & 13 & 0 \\
records excluded & 7 & 0 \\
records included & 6 & 0 \\
Level 1 clinical evidence & 6 & 1 \\
(systematic review, meta analysis) & & 3 \\
Level 2 clinical evidence (RCT) & 12 & \\
Level $\geq 3$ clinical evidence & & 0 \\
trials other than RCT & 0 & 0 \\
case reports & 0 & 0 \\
pharmacokinetic studies & 6 & 4 \\
Economic evidence & 0 & 0 \\
Total records included & 24 & \\
\hline For definition of levels of evidence, see Editorial lnformation on inside back cover. & & \\
RCT, randomized controlled trial. & & \\
\hline
\end{tabular}

Online abstracts from the following congresses were also searched in December, 2005 using the search terms "lumiracoxib OR Prexige": 
- ACR, all conferences from 2002 to 2005, http://www.rheumatology.org/annual/abstracts/search.asp

- EULAR, all conferences from 2004 to 2005, http://www.abstracts2view.com/eular/

- Osteoarthritis Research Society International (OARSI) 9th World Congress, December 2-5, 2004,

http://www.oarsi.org/04Abstracts/Final_OARSI04_Abstracts.pdf

A total of 32 abstracts were identified (Table 1). Twenty-eight abstracts were excluded because they were animal studies $(n=3)$, in-vitro studies $(n=1)$, were in indications other than $O A(n=3)$, were nonrelevant pharmacokinetic/pharmacodynamic studies $(n=2)$, did not cite data $(n=1)$, or were abstracts that duplicated results from $O A$ studies which have since been published in full $(n=18)$. One abstract was level 1 evidence (pooled analysis), and all other abstracts were of level 2 evidence.

PubMed searches were updated on January 27, 2006 and again on May 14, 2007. A total of 13 new records were identified, of which seven were excluded for the following reasons: in-vivo study $(n=1)$, animal study $(n=1)$, review article $(n=5)$. Six articles remained, and were included (Table 1).

\section{Disease overview}

$\mathrm{OA}$ is the most common form of arthritis, which gradually damages the cartilage lining the joints (and the underlying bone) primarily in the hands, knees, hips, and spine. The four main risk factors for OA are age, family history, obesity, and joint trauma (WHO 2003). It is estimated that symptomatic OA affects $9.6 \%$ of men and $18 \%$ of women aged $\geq 60$ years worldwide, and is more prevalent in Europe and the US than in other parts of the world (Woolf \& Pfleger 2003). Of the 115.9 million US adults aged $\geq 35$ years, 24.3 million (21\%) have OA (Singh et al. 2002). Globally, approximately $40 \%$ of individuals over 70 years of age suffer from OA of the knee (WHO 2003).

The prevalence of $\mathrm{OA}$ is expected to increase due to the increasing proportion of the elderly in the population (Reginster 2002), and it is expected to be the fourth leading cause of disability by 2020 (Woolf \& Pfleger 2003). The increasing burden of musculoskeletal disorders, such as OA, has been recognized by the United Nations and the World Health Organization (WHO), which has endorsed the Bone and Joint Decade 2000-2010 initiative, with the aim of increasing awareness of these conditions (Woolf \& Pfleger 2003).

The symptoms of OA include joint pain, stiffness, and a reduced ability to perform normal daily activities, which result in a decreased quality of life. Approximately $80 \%$ of patients with OA have limitation of movement and $25 \%$ cannot perform their major daily activities (WHO 2003). Indeed, musculoskeletal conditions such as OA have been associated with a poorer quality of life than CV, chronic respiratory, and GI conditions (Reginster 2002).

\section{Economic burden of $O A$}

In the US alone, of the \$US214.9 billion in total costs for musculoskeletal disorders in 1995, \$US82.4 billion was associated with various forms of arthritis (the most prevalent being OA and RA) (WHO 2003). In addition, the economic burden of musculoskeletal disorders in Canada has been reported to be close to that of cancer (Badley 1995).

The direct and indirect costs of OA place a considerable burden both on the individual and society. A Canadian study estimating the direct and indirect arthritis-attributable costs to individuals with disabling hip and/or knee OA demonstrated that $80 \%$ of costs incurred were for time lost from employment and leisure by the patient and unpaid informal caregivers. Costs increased with worsening health status and greater OA severity (Gupta et al. 2005). In addition, a European retrospective study in patients with $O A$ of the knee reported that direct costs over a period of 12 months (2000-2001) were €934/ patient per year (including $€ 146$ on therapy, of which $42 \%$ was spent on drugs), and indirect costs were $€ 1236 /$ patient per year (31\% due to loss of productivity of patients and $60 \%$ due to informal care provided by primary caregivers) (Leardini et al. 2004).

\section{Pathophysiology of OA}

Prostaglandins cause the swelling and pain associated with OA (Mitchell \& Warner 1999). The cyclooxygenase enzymes play a key role in the production of prostaglandins by converting arachidonic acid to prostaglandin $\mathrm{H}_{2}\left(\mathrm{PGH}_{2}\right)$, which is then metabolized by specific isomerases to tissue-specific prostanoids [e.g. $\mathrm{PGl}_{2}$ (prostacyclin) and $\mathrm{TXA}_{2}$ (thromboxane)] (Fig. 1). To date, two isoforms of the cycloxoygenase enzyme, COX-1 and COX-2, have been identified. COX-1 is produced constitutively in several tissues including the Gl tract, endothelial cells, kidney, and platelets, whereas COX-2 is expressed constitutively in some tissues (e.g. kidney) and is also inducible (Dubois et al. 1998; Warner \&

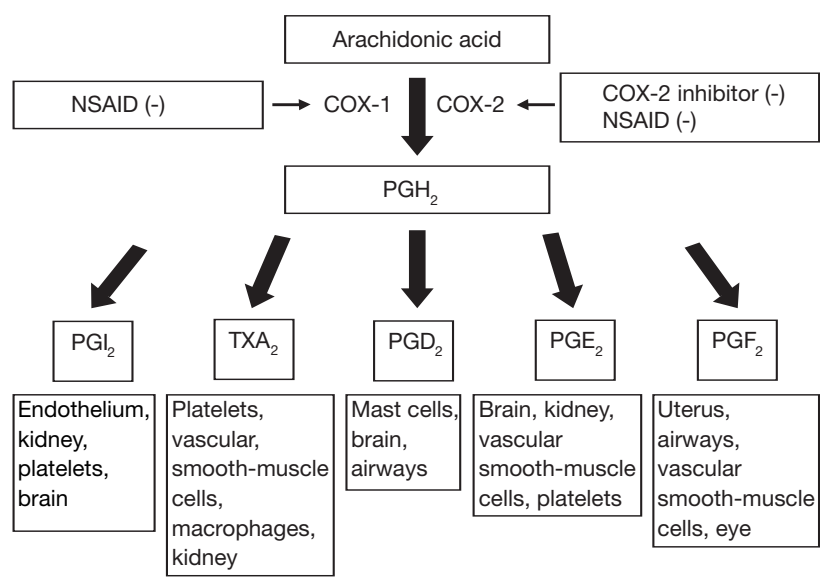

Fig. 1 | The cyclooxygenase pathway (reproduced with permission from Fitzgerald \& Patrono 2001). Arachidonic acid is converted by cyclooxygenase (COX)- 1 and COX-2 to the intermediate prostaglandin $\mathrm{H}_{2}\left(\mathrm{PGH}_{2}\right)$. $\mathrm{PGH}_{2}$ is converted by tissue-specific isomerases to multiple prostanoids; prostacyclin $\left(\mathrm{PGl}_{2}\right)$, thromboxane $A_{2}\left(T X A_{2}\right)$, prostaglandin $D_{2}\left(P G D_{2}\right)$, prostaglandin $E_{2}$ $\left(\mathrm{PGE}_{2}\right)$, and prostaglandin $\mathrm{F}_{2}\left(\mathrm{PGF}_{2}\right)$. These prostanoids exert prominent effects via cell-membrane receptors 
Mitchell 2004). Through its production of gastroprotective prostaglandin $E_{2}\left(P_{G}\right)$, the constituent COX-1 enzyme is recognized as being protective of the Gl system. Cytokines and growth factors increase the expression of COX-2 at inflammatory sites. Therefore, the COX-2 enzyme primarily mediates pain and inflammation (Warner \& Mitchell 2004).

\section{Current therapy options}

Currently, there is no known cure for OA or means of preventing it. The main goals of OA management are to control pain and to improve function and quality of life, while avoiding adverse side effects. Pharmacologic treatments for OA include: analgesics such as acetaminophen (paracetamol), which relieve pain but do not reduce inflammation; NSAIDs, which are both analgesic and antiinflammatory; injectable corticosteroids; and symptomatic slow-acting drugs for OA such as glucosamine sulfate, chondroitin sulfate, and hyaluronic acid. Nonpharmacologic treatments such as patient education, diet, exercise, and the use of insoles may also improve the symptoms of OA. Surgical treatment (i.e. prosthetic joint replacement) is generally only used when there is significant joint damage and all other treatment options have failed.

NSAIDs relieve pain and inflammation by blocking the action of the COX-2 enzyme. There are currently two types of NSAIDs: nonselective NSAIDs suppress both the COX-1 and COX-2 enzymes to varying degrees; COX-2 inhibitors (i.e. selective NSAIDs) suppress the COX-2 enzyme only (Fitzgerald \& Patrono 2001).

\section{Nonselective NSAIDs}

Nonselective NSAIDs such as naproxen, ibuprofen, diclofenac, and nabumetone, have been available on the market for many years. A major disadvantage of nonselective NSAIDs is their potential to cause Gl side effects by inhibition of COX-1 and topical toxicity (Lazzaroni \& Porro 2004). For example, nonselective NSAIDs increase the risk of serious ulcer complications (bleeding, perforation, obstruction), hospitalization, surgery, and death (Singh et al. 1997; Wolfe et al. 1999). In the 1980s, Gl complications caused by nonselective NSAID use was estimated to cause approximately 16000 deaths and 100000 hospitalizations each year in the US alone.

Risk factors for NSAID-associated upper GI complications include use of multiple NSAIDs (e.g. NSAID and aspirin), high doses of NSAIDs, prior ulcer complications, advanced age, and concomitant corticosteroid or anticoagulant use (Scheiman \& Fendrick 2005).

Approximately $50 \%$ of patients who regularly take nonselective NSAIDs have gastric erosions, and $15-30 \%$ have ulcers when examined by endoscopy. Clinical upper Gl events may occur in $3-4.5 \%$ of patients taking NSAIDs, and serious complicated events develop in approximately $1.5 \%$ (Laine 2001). These GI adverse events often lead to discontinuation of treatment, and may have an important impact on the patient's quality of life and healthcare costs. Therefore, the human and economic costs of these complications could be reduced by using medications which reduce their incidence.
In order to reduce the incidence of Gl complications nonselective NSAIDs may be used in combination with gastroprotective agents such as misprosotol, $\mathrm{H}_{2}$ receptor antagonists, and proton pump inhibitors (PPIs), which add to the total cost of therapy. But patient compliance to misprosotol is limited by its side effects (e.g. diarrhea and abdominal cramps), and $\mathrm{H}_{2}$ receptor antagonists do not offer significant protection against gastric ulcers (Singh \& Triadafilopoulos 2005). In comparison to these other treatments PPIs are better tolerated. Eradication of Helicobacter pylori intestinal infection has also been shown to reduce peptic ulcer incidence in patients receiving NSAIDs. However, it was less effective at preventing NSAID-associated ulcers than treatment with a PPI (Vergara et al. 2005). Evidence suggests that $H$. pylori infection and NSAID use are independent risk factors for Gl complications (Huang et al. 2002).

COX-1 also plays a role in the synthesis of prostanoids which regulate renal function. It is well established that nonselective NSAIDs have adverse effects on the kidney, and may cause peripheral edema and hypertension (Gambaro \& Perazella 2003). Data from two meta analyses have shown that nonselective NSAIDs increase blood pressure (BP), although the effect varied depending upon the NSAID used (Pope et al. 1993; Johnson et al. 1994). In addition, two prospective studies have recently reported conflicting data regarding the increased risk of hypertension with the use of nonselective NSAIDs. A study in women demonstrated an increased risk of hypertension with high daily doses of acetaminophen and ibuprofen (Forman et al. 2005). In contrast, a study in men did not demonstrate an increased risk with these drugs (Kurth et al. 2005).

There is limited evidence that nonselective NSAIDs are associated with an increased risk of CV events. Data from an observational study of 367 general practices in the UK between 2000 and 2004 has suggested that the use of diclofenac and ibuprofen is associated with an increased risk of myocardial infarction (MI) (Hippisley-Cox \& Coupland 2005).

\section{Selective COX-2 inhibitors}

The COX-2 inhibitors are a new generation of NSAIDs. These drugs were developed to selectively inhibit the COX-2 enzyme with the aim of stopping the production of inflammatory prostaglandins without inhibiting the production of prostaglandins that protect the stomach and intestine. Therefore, these selective COX-2 inhibitors were proposed to provide comparable efficacy to that of nonselective NSAIDs, but with a lower incidence of $\mathrm{Gl}$ adverse effects. Several COX-2 inhibitors have been developed for the treatment of OA including etodolac and meloxicam which are relatively COX-2 selective, and rofecoxib, celecoxib, valdecoxib, etoricoxib, and lumiracoxib which are highly COX-2 selective.

A review of the efficacy and safety of the COX-2 inhibitors is beyond the scope of this article. Therefore, only the major issues (i.e. their $\mathrm{Gl}$ effects and potential CV risk) currently surrounding the COX-2 inhibitors will be summarized. 


\section{Gl effects}

There is conflicting data regarding the Gl benefit of COX-2 inhibitors compared with the nonselective NSAIDs. Large-scale GI safety studies with rofecoxib [Vioxx Gastrointestinal Outcomes Research (VIGOR) trial] and celecoxib [Celecoxib Long-term Arthritis Safety Study (CLASS)] have demonstrated that these COX-2 inhibitors reduce the incidence of $\mathrm{Gl}$ adverse events compared with nonselective NSAIDs (Bombardier et al. 2000; Silverstein et al. 2000). However, unpublished data from the CLASS trial have shown that the Gl advantage seen with celecoxib at 6 months was not evident after 13 months (FDA 2001). In addition, a review of data from an observational study of 367 general practices in the UK suggested that rofecoxib did not offer a GI benefit over the nonselective NSAIDs (HippisleyCox et al. 2005).

\section{CV profile}

COX-2 inhibitors appear to suppress the production of prostacyclin, a vasodilator that inhibits platelet aggregation and thereby reduces blood clotting. It has been hypothesized that selective inhibition of COX-2 may alter the balance between the antithrombotic prostacyclin and prothrombotic $\mathrm{TXA}_{2}$, selectively inhibiting the production of prostacyclin and allowing the effects of thromboxane to predominate, thereby promoting a prothrombotic state (Jones 2005).

Emerging data have raised concerns over an increased risk of CV events with COX-2 inhibitors. Data from the cancer prevention trial Adenomatous Polyp Prevention on Vioxx (APPROVe) suggested that patients receiving rofecoxib had a statistically significant increase in heart attacks and other adverse CV events such as strokes (Bresalier et al. 2005). It is not evident whether the increased risk of $\mathrm{CV}$ events seen with rofecoxib is a class effect. However, the Adenoma Prevention with Celecoxib (APC) trial also demonstrated 2.5 times the rate of a major CV event among patients taking celecoxib compared with placebo (Solomon et al. 2005), and there is some evidence for increased $\mathrm{CV}$ risk with another COX-2 inhibitor, ertoricoxib (Aldington et al. 2005). A systematic review of the clinical literature (from 1996 to March 2005) attempted to determine the relative thromboembolic risks of COX-2 inhibitors and concluded that each COX-2 inhibitor has a unique CV risk profile (Jones 2005). The author noted that the risk of a CV event may depend upon the agent chosen and its COX-2-specific isoform-binding affinity, the $\mathrm{CV}$ risk profile of the patient, and the dose and duration of the drug use.

\section{Renal function}

In addition to the role of COX-1, COX-2 is also involved in the synthesis of prostaglandins which play a role in modulating renal function. Consequently COX-2 inhibitors have been associated with similar renal adverse effects to those seen with nonselective NSAIDs. Therefore, the COX-2 inhibitors may not offer a clinical advantage over nonselective NSAIDs with regards to avoiding or exacerbating edema, hypertension, and congestive heart failure.
The COX-2 inhibitors rofecoxib and celecoxib have been associated with slightly higher BP compared with nonselective NSAIDs (Whelton et al. 2001; Whelton et al. 2002; Aw et al. 2005). A retrospective cohort study demonstrated that more elderly patients with a previous episode of congestive heart failure prescribed celecoxib survived or remained free of recurrent congestive heart failure than those prescribed either rofecoxib or nonselective NSAIDs. These data suggest that differences may exist between the classes of nonselective NSAIDs and COX-2 inhibitors, and between COX-2 inhibitors (Hudson et al. 2005).

\section{Current treatment guidelines}

It is recommended that all pharmacologic agents should be considered in addition to nonpharmacologic measures for the treatment of OA. The first drug of choice for the treatment of inflammation and pain in patients with $O A$ is acetaminophen, which when using the appropriate dosage may be taken safely over the long term, and is inexpensive compared with prescription NSAIDs (ACR 2000; Pendleton et al. 2000; NICE 2001). Alternatively, US guidelines for the medical management of OA of the hip and knee suggest that NSAIDs or joint aspiration in combination with intraarticular injection of glucocorticoids may be considered as first-line pharmacologic therapy in patients with moderate to severe pain, and with signs of joint inflammation (ACR 2000). In patients with risk factors for serious upper GI events and renal toxicity, US guidelines recommend the use of COX-2 selective inhibitors, or nonselective NSAIDs in combination with a gastroprotective agent (misoprostol or a PPI) (ACR 2000).

European (EULAR) evidence-based guidelines for the management of knee OA state that there is good evidence [from at least one randomized, controlled trial $(\mathrm{RCT})]$ to suggest that NSAIDs are more efficacious in providing pain relief than acetaminophen (Pendleton et al. 2000). In patients who fail to achieve adequate symptomatic relief with acetaminophen, European guidelines recommend the use of oral or topical NSAIDs (Pendleton et al. 2000).

Current NICE guidelines on the use of COX-2 inhibitors (celecoxib, rofecoxib, meloxicam, and etodolac) do not recommend the routine use of COX-2 selective inhibitors in patients with OA (NICE 2001). These guidelines state that they should only be used instead of nonselective NSAIDs in patients at high-risk of developing serious $\mathrm{Gl}$ adverse effects (patients aged $\geq 65$ years, using concomitant medication that increases the likelihood of upper Gl adverse events, serious comorbidity present, or requiring prolonged use of maximum recommended doses of NSAIDs). It is assumed that these recommendations are based upon the improved cost effectiveness reported with COX-2 inhibitors in this high-risk population, compared with nonselective NSAIDs.

Since the publication of these guidelines, rofecoxib and valdecoxib have been withdrawn from all markets (Table 2). A joint meeting of the Arthritis Advisory Committee and the Drug Safety and Risk Management Advisory Committee reviewed the safety of all COX-2 inhibitors (FDA 2005a). They concluded that the risk versus benefit of COX-2 inhibitors favored their continued use in the US and that although all NSAIDs impose similar CV risks, some agents 
Table 4 | Effect of lumiracoxib on pain intensity and patient's/physician's global assessment of disease activity in patients with OA of the knee, hip or hand (all published level 2 evidence is cited as original source; level 2 evidence only published within the systematic review of Berenbaum et al. 2005 is cited as such)

\begin{tabular}{|c|c|c|c|c|c|}
\hline \multirow[t]{2}{*}{ Reference } & \multirow{2}{*}{$\begin{array}{l}\text { Design and } \\
\text { population }\end{array}$} & \multirow{2}{*}{$\begin{array}{l}\text { Treatment } \\
\text { (no. patients) }\end{array}$} & \multicolumn{3}{|c|}{ Outcome [mean change from baseline to end of study (100 mm VAS)] } \\
\hline & & & $\begin{array}{l}\text { Pain intensity in } \\
\text { target knee (mm) }\end{array}$ & $\begin{array}{l}\text { Patient's global } \\
\text { assessment of } \\
\text { disease activity }(\mathrm{mm})\end{array}$ & $\begin{array}{l}\text { Physician's global } \\
\text { assessment of } \\
\text { disease activity }(\mathrm{mm})\end{array}$ \\
\hline \multicolumn{6}{|l|}{ 4-week studies } \\
\hline Berenbaum et al. $2005^{a}$ & $\begin{array}{l}\text { Hip or knee OA } \\
\mathrm{db}, \mathrm{RCT}\end{array}$ & $\begin{array}{l}100 \text { mg LX od (122) } \\
\text { PL (122) }\end{array}$ & $\begin{array}{l}-24.6 \\
-16.8\end{array}$ & $\begin{array}{l}\mathrm{LX}-\mathrm{PL} \text { difference in LSM } \\
-8.8 ; P=0.001\end{array}$ & NR \\
\hline Schnitzer et al. $2004 a$ & $\begin{array}{l}\text { Hip or knee OA } \\
\text { Phase II dose- } \\
\text { finding study } \\
\text { db, RCT }\end{array}$ & $\begin{array}{l}50 \text { mg LX bid (98) } \\
100 \text { mg LX bid (96) } \\
200 \text { mg LX bid (99) } \\
400 \text { mg LX od (99) } \\
75 \text { mg diclofenac bid (94) } \\
\text { PL (97) }\end{array}$ & $\begin{array}{l}\text { Mean overall scores at } \\
\text { baseline of } 64.7-67.0 \\
\text { were reduced to } 33.7- \\
38.4 \mathrm{~mm} \text { with all doses } \\
\text { of } \mathrm{LX} \text { and diclofenac, } \\
\text { and from } 67.9 \text { to } 50.2 \\
\text { with placebo }\end{array}$ & $\begin{array}{l}-24.3 \\
-24.2 \\
-26.5 \\
-28.1 \\
-27.8 \\
-12.5\end{array}$ & $\begin{array}{l}-21.8 \\
-23.2 \\
-27.0 \\
-26.5 \\
-23.8 \\
-13.1\end{array}$ \\
\hline Grifka et al. 2004 & $\begin{array}{l}\text { Hand OA } \\
\mathrm{db}, \mathrm{mc}, \mathrm{RCT}\end{array}$ & $\begin{array}{l}200 \text { mg LX od (205) } \\
400 \text { mg LX od (193) } \\
\text { PL (196) }\end{array}$ & $\begin{array}{l}-28.0 \\
-30.0 \\
-19.3\end{array}$ & $\begin{array}{l}-16.3 \\
-20.9 \\
-9.4\end{array}$ & $\begin{array}{l}-17.8 \\
-18.7 \\
-12.5\end{array}$ \\
\hline \multicolumn{6}{|l|}{ 13-week studies } \\
\hline Lehmann et al. 2005 & $\begin{array}{l}\text { Knee OA } \\
\mathrm{db}, \mathrm{dd}, \mathrm{mc}, \mathrm{RCT}\end{array}$ & $\begin{array}{l}100 \mathrm{mg} \text { LX od (420) } \\
100 \mathrm{mg} \text { LX od }+200 \mathrm{mg} \text { loading } \\
\text { dose LX od for first } 2 \text { wk (420) } \\
200 \mathrm{mg} \text { CX od (420) } \\
\text { PL (424) }\end{array}$ & $\begin{array}{l}-26.8 \\
-26.2 \\
-26.6 \\
-21.4\end{array}$ & $\begin{array}{l}-25.1 \\
-21.9 \\
-22.9 \\
-18.9\end{array}$ & $\begin{array}{l}-26.3 \\
-25.0 \\
-25.4 \\
-20.4\end{array}$ \\
\hline Sheldon et al. 2005 & $\begin{array}{l}\text { Knee OA } \\
\mathrm{db}, \mathrm{dd}, \mathrm{mc}, \mathrm{RCT}\end{array}$ & $\begin{array}{l}100 \text { mg LX od (391) } \\
100 \text { mg LX od + } 200 \text { mg loading } \\
\text { dose LX od for first } 2 \text { wk (385) } \\
200 \text { mg CX od (393) } \\
\text { PL (382) }\end{array}$ & $\begin{array}{l}-25.1 \\
-25.9 \\
-24.1 \\
-18.1\end{array}$ & $\begin{array}{l}-23.2 \\
-24.6 \\
-19.5 \\
-13.4\end{array}$ & $\begin{array}{l}-25.5 \\
-26.9 \\
-22.2 \\
-15.7\end{array}$ \\
\hline Tannenbaum et al. 2004 & $\begin{array}{l}\text { Knee OA } \\
\mathrm{db}, \mathrm{dd}, \mathrm{mc}, \mathrm{RCT}\end{array}$ & $\begin{array}{l}200 \mathrm{mg} \text { LX od }(487) \\
400 \mathrm{mg} \text { LX od }(491) \\
200 \mathrm{mg} \mathrm{CX} \text { od }(481) \\
\text { PL (243) }\end{array}$ & $\begin{array}{l}-26.0 \\
-27.4 \\
-25.2 \\
-19.8\end{array}$ & $\begin{array}{l}-23.2 \\
-24.1 \\
-22.4 \\
-15.7\end{array}$ & $\begin{array}{l}-23.0 \\
-23.6 \\
-22.4 \\
-18.0\end{array}$ \\
\hline Fleischmann et al. 2005 & $\begin{array}{l}\text { Knee OA } \\
\mathrm{db}, \mathrm{dd}, \mathrm{mc}, \mathrm{RCT}\end{array}$ & $\begin{array}{l}200 \text { mg LX od (462) } \\
400 \text { mg LX od (463) } \\
200 \text { mg CX od (444) } \\
\text { PL (231) }\end{array}$ & $\begin{array}{l}-28.7 \\
-29.7 \\
-27.4 \\
-21.3\end{array}$ & $\begin{array}{l}-25.3 \\
-25.8 \\
-24.5 \\
-16.1\end{array}$ & $\begin{array}{l}-27.2 \\
-26.7 \\
-24.5 \\
-18.3\end{array}$ \\
\hline Berenbaum et al. 2005 & $\begin{array}{l}\text { Hip OA } \\
\text { RCT }\end{array}$ & $\begin{array}{l}400 \mathrm{mg} \mathrm{LX} \text { od (205) } \\
25 \mathrm{mg} \mathrm{RX} \text { od (102) } \\
\text { PL (204) }\end{array}$ & $\begin{array}{l}-21.8 \\
-25.0 \\
-15.1\end{array}$ & $\begin{array}{l}-18.3 \\
-21.8 \\
-11.6\end{array}$ & $\begin{array}{l}\mathrm{NR} \\
\mathrm{NR} \\
\mathrm{NR}\end{array}$ \\
\hline \multicolumn{6}{|l|}{ 52-week studies } \\
\hline Berenbaum et al. $2005^{b}$ & $\begin{array}{l}\text { Knee OA } \\
\text { 39-wk extension } \\
\text { of Tannenbaum } \\
\text { et al. } 2004\end{array}$ & $\begin{array}{l}200 \mathrm{mg} \text { LX od (352) } \\
400 \mathrm{mg} \text { LX od (358) } \\
200 \mathrm{mg} \mathrm{CX} \text { od }(348) \\
200 \mathrm{mg} \text { LX od }(56)^{\mathrm{c}} \\
400 \mathrm{mg} \text { LX od }(59)^{\mathrm{C}} \\
200 \mathrm{mg} \mathrm{CX} \text { od }(56)^{\mathrm{c}}\end{array}$ & $\begin{array}{l}-28.1 \\
-28.7 \\
-28.3 \\
-3.3 \\
-5.6 \\
-4.3\end{array}$ & $\begin{array}{l}-25.2 \\
-24.6 \\
-26.1 \\
-5.2 \\
-5.8 \\
-9.2\end{array}$ & $\begin{array}{l}\text { NR } \\
\text { NR } \\
\text { NR } \\
\text { NR } \\
\text { NR } \\
\text { NR }\end{array}$ \\
\hline $\begin{array}{l}\text { Pavelka et al. } 2005 \\
\text { (abstract) }\end{array}$ & $\begin{array}{l}\text { Knee OA } \\
\text { 39-wk extension } \\
\text { of Lehmann et al. } \\
2005\end{array}$ & $\begin{array}{l}100 \text { mg LX od (659) } \\
200 \text { mg CX od (327) } \\
100 \text { mg LX od (176) } \\
200 \text { mg CX od (130) }\end{array}$ & $\begin{array}{l}\text { LSM for LX and CX } \\
\text { were similar at study } \\
\text { end: } 33.2 \mathrm{~mm} \text { and } \\
31.0 \mathrm{~mm} \text {, respectively }\end{array}$ & $\begin{array}{l}\text { LSM for LX and CX were } \\
\text { similar at study end: } \\
36.6 \mathrm{~mm} \text { and } 34.2 \mathrm{~mm} \text {, } \\
\text { respectively }\end{array}$ & NR \\
\hline $\begin{array}{l}\text { Schnitzer et al. } 2004 \mathrm{~b}, \\
\text { Grifka et al. } 2005 \\
\text { (abstract) }\end{array}$ & $\begin{array}{l}\text { Hip, knee, or } \\
\text { hand OA }\end{array}$ & $\begin{array}{l}400 \text { mg LX od (9117) } \\
500 \text { mg naproxen bid (4730) } \\
800 \text { mg ibuprofen tid (4397) }\end{array}$ & $\begin{array}{l}\text { Reduction in pain } \\
\text { intensity with LX was } \\
\text { similar to that of } \\
\text { NSAIDs [62.7\% vs } \\
61.9 \% \text { (NSAID } \\
\text { combined data); } \\
P=0.107 \text { ], regardless of } \\
\text { the joint affected }\end{array}$ & 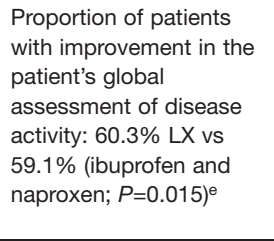 & $\begin{array}{l}\text { Proportion of patients } \\
\text { with improvement in the } \\
\text { physician's global } \\
\text { assessment of disease } \\
\text { activity: } 59.2 \% \text { LX vs } \\
58.3 \% \text { (ibuprofen and } \\
\text { naproxen; } P=0.023 \text { ) }\end{array}$ \\
\hline
\end{tabular}


Table 5 | Effect of lumiracoxib on WOMAC/AUSCAN scores in patients with OA of the knee, hip, or hand (all published level 2 evidence is cited as original source; level 2 evidence only published within the systematic review of Berenbaum et al. 2005 is cited as such)

\begin{tabular}{|c|c|c|c|c|c|c|}
\hline \multirow[t]{2}{*}{ Reference } & \multirow[t]{2}{*}{$\begin{array}{l}\text { Design and } \\
\text { population }\end{array}$} & \multirow[t]{2}{*}{$\begin{array}{l}\text { Treatment } \\
\text { (no. patients) }\end{array}$} & \multicolumn{4}{|c|}{$\begin{array}{l}\text { Outcome (mean change from baseline to end of study) } \\
\text { WOMAC scores (AUSCAN for OA of hand) }\end{array}$} \\
\hline & & & Total score & Pain subscale & DPDA subscale & Stiffness subscale \\
\hline \multicolumn{7}{|l|}{ 4-week studies } \\
\hline Berenbaum et al. $2005^{a}$ & $\begin{array}{l}\text { Hip or knee OA } \\
\mathrm{db}, \mathrm{RCT}\end{array}$ & $\begin{array}{l}100 \mathrm{mg} \text { LX od (122) } \\
\mathrm{PL}(122)\end{array}$ & $\begin{array}{l}\text { LX-PL difference in LSM } \\
-4.5 ; P=0.021\end{array}$ & NR & NR & NR \\
\hline Schnitzer et al. 2004a & $\begin{array}{l}\text { Hip or knee OA } \\
\text { Phase II dose- } \\
\text { finding study } \\
\mathrm{db}, \mathrm{RCT}\end{array}$ & $\begin{array}{l}50 \text { mg LX bid (98) } \\
100 \text { mg LX bid (96) } \\
200 \text { mg LX bid (99) } \\
400 \text { mg LX od (99) } \\
75 \text { mg diclofenac bid (94) } \\
\text { PL (97) }\end{array}$ & $\begin{array}{l}\text { NR } \\
\text { NR } \\
\text { NR } \\
\text { NR } \\
\text { NR } \\
\text { NR }\end{array}$ & $\begin{array}{l}-3.4 \\
-2.8 \\
-3.2 \\
-3.8 \\
-3.3 \\
-1.5\end{array}$ & $\begin{array}{l}-10.3 \\
-7.9 \\
-10.7 \\
-13.0 \\
-11.1 \\
-5.4\end{array}$ & $\begin{array}{l}-1.7 \\
-1.3 \\
-1.5 \\
-1.6 \\
-1.3 \\
-0.7\end{array}$ \\
\hline Grifka et al. 2004 & $\begin{array}{l}\text { Hand OA } \\
\mathrm{db}, \mathrm{mc}, \mathrm{RCT}\end{array}$ & $\begin{array}{l}200 \text { mg LX od (205) } \\
400 \text { mg LX od (193) } \\
\text { PL (196) }\end{array}$ & $\begin{array}{l}-7.7 \\
-10.5 \\
-5.6\end{array}$ & $\begin{array}{l}-3.0 \\
-3.9 \\
-2.1\end{array}$ & $\begin{array}{l}-4.3 \\
-6.0 \\
-3.1\end{array}$ & $\begin{array}{l}-0.6 \\
-0.7 \\
-0.4\end{array}$ \\
\hline \multicolumn{7}{|l|}{ 13-week studies } \\
\hline Lehmann et al. 2005 & $\begin{array}{l}\text { Knee OA } \\
\mathrm{db}, \mathrm{dd}, \mathrm{mc} \text {, } \\
\text { RCT }\end{array}$ & $\begin{array}{l}100 \mathrm{mg} \text { LX od (420) } \\
100 \mathrm{mg} \text { LX od }+200 \mathrm{mg} \text { loading } \\
\text { dose LX od for first } 2 \text { wk (420) } \\
200 \mathrm{mg} \text { CX od (420) } \\
\text { PL (424) }\end{array}$ & $\begin{array}{l}-15.2 \\
-14.8 \\
-14.7 \\
-11.3\end{array}$ & $\begin{array}{l}-3.4 \\
-3.2 \\
-3.4 \\
-2.5\end{array}$ & $\begin{array}{l}-10.5 \\
-10.4 \\
-10.3 \\
-8.0\end{array}$ & $\begin{array}{l}-1.3 \\
-1.1 \\
-1.1 \\
-0.9\end{array}$ \\
\hline Sheldon et al. 2005 & $\begin{array}{l}\text { Knee OA } \\
\mathrm{db}, \mathrm{dd}, \mathrm{mc} \text {, } \\
\text { RCT }\end{array}$ & $\begin{array}{l}100 \mathrm{mg} \text { LX od (391) } \\
100 \mathrm{mg} \text { LX od }+200 \mathrm{mg} \text { loading } \\
\text { dose LX od for first } 2 \text { wk (385) } \\
200 \mathrm{mg} \text { CX od (393) } \\
\text { PL (382) }\end{array}$ & $\begin{array}{l}-16.9 \\
-17.2 \\
-15.6 \\
-9.5\end{array}$ & $\begin{array}{l}-3.6 \\
-3.7 \\
-3.4 \\
-2.3\end{array}$ & $\begin{array}{l}-11.9 \\
-12.0 \\
-10.8 \\
-6.3\end{array}$ & $\begin{array}{l}-1.5 \\
-1.5 \\
-1.4 \\
-0.9\end{array}$ \\
\hline Tannenbaum et al. 2004 & $\begin{array}{l}\text { Knee OA } \\
\mathrm{db}, \mathrm{dd}, \mathrm{mc} \text {, } \\
\text { RCT }\end{array}$ & $\begin{array}{l}200 \mathrm{mg} \text { LX od }(487) \\
400 \mathrm{mg} \text { LX od }(491) \\
200 \mathrm{mg} \mathrm{CX} \text { od }(481) \\
\mathrm{PL}(243)\end{array}$ & $\begin{array}{l}-14.1 \\
-14.1 \\
-13.4 \\
-9.4\end{array}$ & $\begin{array}{l}-3.2 \\
-3.2 \\
-3.1 \\
-2.4\end{array}$ & $\begin{array}{l}-9.8 \\
-9.7 \\
-9.2 \\
-6.2\end{array}$ & $\begin{array}{l}-1.2 \\
-1.2 \\
-1.2 \\
-0.9\end{array}$ \\
\hline Fleischmann et al. 2005 & $\begin{array}{l}\text { Knee OA } \\
\mathrm{db}, \mathrm{dd}, \mathrm{mc} \text {, } \\
\text { RCT }\end{array}$ & $\begin{array}{l}200 \mathrm{mg} \text { LX od (462) } \\
400 \mathrm{mg} \text { LX od (463) } \\
200 \mathrm{mg} \mathrm{CX} \text { od }(444) \\
\mathrm{PL}(231)\end{array}$ & $\begin{array}{l}-17.8 \\
-16.9 \\
-16.0 \\
-9.3\end{array}$ & $\begin{array}{l}-3.7 \\
-3.7 \\
-3.5 \\
-2.3\end{array}$ & $\begin{array}{l}-12.5 \\
-11.7 \\
-11.0 \\
-6.1\end{array}$ & $\begin{array}{l}-1.6 \\
-1.4 \\
-1.4 \\
-0.9\end{array}$ \\
\hline Berenbaum et al. 2005 & $\begin{array}{l}\text { Hip OA } \\
\text { RCT }\end{array}$ & $\begin{array}{l}400 \mathrm{mg} \text { LX od (205) } \\
25 \mathrm{mg} \mathrm{RX} \text { od (102) } \\
\text { PL (204) }\end{array}$ & $\begin{array}{l}-11.4 \\
-14.6 \\
-8.6\end{array}$ & $\begin{array}{l}-2.7 \\
-3.1 \\
-2.1\end{array}$ & $\begin{array}{l}\text { NR } \\
\text { NR } \\
\text { NR }\end{array}$ & $\begin{array}{l}\text { NR } \\
\text { NR } \\
\text { NR }\end{array}$ \\
\hline 52-week studies & & & & & & \\
\hline Berenbaum et al. $2005^{b}$ & $\begin{array}{l}\text { Knee OA } \\
\text { 39-wk extension } \\
\text { of Tannenbaum } \\
\text { et al. } 2004\end{array}$ & $\begin{array}{l}200 \mathrm{mg} \text { LX od (352) } \\
400 \mathrm{mg} \text { LX od }(358) \\
200 \mathrm{mg} \mathrm{CX} \text { od }(348) \\
200 \mathrm{mg} \mathrm{LX} \text { od }(56)^{\mathrm{c}} \\
400 \mathrm{mg} \mathrm{LX} \text { od }(59)^{\mathrm{c}} \\
200 \mathrm{mg} \mathrm{CX} \text { od }(56)^{\mathrm{c}}\end{array}$ & $\begin{array}{l}-16.1 \\
-16.4 \\
-15.5 \\
-3.5 \\
-2.1 \\
-5.9\end{array}$ & $\begin{array}{l}-3.6 \\
-3.7 \\
-3.4 \\
-0.4 \\
-0.5 \\
-1.5\end{array}$ & $\begin{array}{l}\text { NR } \\
\text { NR } \\
\text { NR } \\
\text { NR } \\
\text { NR } \\
\text { NR }\end{array}$ & $\begin{array}{l}\text { NR } \\
\text { NR } \\
\text { NR } \\
\text { NR } \\
\text { NR } \\
\text { NR }\end{array}$ \\
\hline $\begin{array}{l}\text { Pavelka et al. } 2005 \\
\text { (abstract) }\end{array}$ & $\begin{array}{l}\text { Knee OA } \\
\text { 39-wk extension } \\
\text { of Lehmann } \\
\text { et al. } 2005\end{array}$ & $\begin{array}{l}100 \text { mg LX od (659) } \\
200 \text { mg CX od (327) } \\
100 \text { mg LX od (176) } \\
200 \text { mg CX od }(130)^{d}\end{array}$ & $\begin{array}{l}\text { LSM for LX and CX were } \\
\text { similar at study end: } \\
33.0 \mathrm{~mm} \text { and } 30.7 \mathrm{~mm} \text {, } \\
\text { respectively }\end{array}$ & NR & NR & NR \\
\hline $\begin{array}{l}\text { aAlso published in abstract fo } \\
\text { bAlso published in abstract fo } \\
\text { c171 patients receiving place } \\
{ }^{d} 306 \text { patients receiving placel } \\
\text { AUSCAN, Australian/Canadia } \\
\text { least squares mean; LX, lumir } \\
\text { WOMAC, Western Ontario an }\end{array}$ & $\begin{array}{l}\text { m (Benevolenskaya } \\
\text { m (Schell et al. } 2003 \\
\text { o during the core } 13 \\
\text { o during the core } 13 \\
\text { OA Hand Index; bid } \\
\text { acoxib; mc, multicent } \\
\text { McMaster Universit }\end{array}$ & $\begin{array}{l}\text { 2003). } \\
\text { k study were re-randomized to activ } \\
\text { ek study were re-randomized to activ } \\
\text { ice daily; CX, celecoxib; db, double- } \\
\text { VR, not reported; OA, osteoarthritis; } \\
\text { DA Index. }\end{array}$ & $\begin{array}{l}\text { eatment during the extensi } \\
\text { eatment during the extensi } \\
\text {; dd, double-dummy; DPD } \\
\text { once daily; PL, placebo; RC }\end{array}$ & $\begin{array}{l}\text { riod. } \\
\text { eriod. } \\
\text { fficulty in perfo } \\
\text { andomized con }\end{array}$ & $\begin{array}{l}\text { daily activities; LS } \\
\text { d trial; RX, rofecoxi }\end{array}$ & k, week; \\
\hline
\end{tabular}


published in full, including the Therapeutic Arthritis Research and Gastrointestinal Event Trial (TARGET) (Schnitzer et al. 2004b; Lehmann et al. 2005; Sheldon et al. 2005), one RCT published in abstract form (Pavelka et al. 2005), and an additional analysis from TARGET published in abstract form (Grifka et al. 2005), has shown that the pain relief [as assessed by pain intensity on a visual analog scale (VAS)] with lumiracoxib is superior to that gained with placebo and similar to that with celecoxib, rofecoxib, ibuprofen, naproxen, and diclofenac in patients with OA of the hand, hip, or knee (Tables 3 and 4). The efficacy of lumiracoxib does not appear to be dose dependent, with the $100 \mathrm{mg}$ dose providing similar efficacy to the $400 \mathrm{mg}$ dose.

Level 1 and level 2 evidence from these studies also shows that lumiracoxib improves patients' and physicians' assessment of disease activity, as determined by a questionnaire (Table 4). The reduction in scores with lumiracoxib were superior to placebo and comparable with celecoxib, rofecoxib, ibuprofen, naproxen, and diclofenac. These findings suggest that lumiracoxib is effective in managing pain relief in patients with $O A$, and has comparable efficacy to other available NSAIDs.

Pain intensity was reduced by lumiracoxib $400 \mathrm{mg} /$ day (four times greater than the recommended dosage for $O A$ ) to a significantly greater extent than placebo and to a comparable degree as celecoxib $200 \mathrm{mg} /$ day (twice the recommended OA dosage) 3 and 5 hours postdose in patients with knee OA (Wittenberg et al. 2006).

\section{Joint stiffness and function}

Level 1 (Berenbaum et al. 2005) and level 2 evidence (Lehmann et al. 2005; Sheldon et al. 2005) demonstrated that lumiracoxib improves joint stiffness and overall function [assessed using the difficulty in performing daily activities (DPDA) and stiffness subscales of the WOMAC questionnaire or the AUSCAN] compared with placebo (Table 5). These improvements were similar to that achieved with diclofenac and celecoxib, and are expected to have a positive impact on the quality of life of the patient.

\section{SF-36 Health Survey}

The SF-36 Health Survey is a validated questionnaire which provides a measure of generic health by eight health profiles including physical functioning and mental health (Ware 1993). SF-36 was used in two RCTs to determine health-related quality of life in patients with $\mathrm{OA}$ receiving lumiracoxib (Fleischmann et al. 2005; Sheldon et al. 2005). The results showed that lumiracoxib and celecoxib were statistically superior to placebo in the SF-36 physical component (data not reported; defined as limitations in physical activity because of health problems) (Fleischmann et al. 2005; Sheldon et al. 2005) and mental health component (defined as psychologic distress and wellbeing) (Sheldon et al. 2005) at week 13.

\section{Responders}

Analyses of response to lumiracoxib treatment were also performed using the Outcome Measures in Clinical Trials-Osteoarthritis Research Society International (OMERACT-OARSI) criteria (Dougados et al. 2000; Pham et al. 2003). Responders to treatment were defined as patients who either had: a) reduction of $\geq 50 \%$ from baseline and an absolute reduction of $\geq 20$ either in OA pain intensity (100 mm VAS) or WOMAC DPDA subscale score (rescaled to $0-100$ ); or b) reduction of $\geq 20 \%$ and an absolute reduction of $\geq 10$ in at least two of the following variables: OA pain intensity (100 mm VAS), WOMAC DPDA subscale score (rescaled to 0-100) or patient's global assessment of disease activity (100 mm VAS). These criteria demonstrated that a significantly greater proportion of patients receiving active treatment were considered responders compared with placebo (data not stated, reported graphically; all $P<0.05$ vs placebo) (Berenbaum et al. 2005; Fleischmann et al. 2005; Lehmann et al. 2005; Sheldon et al. 2005).

Table 6 | Patient satisfaction (\%) with lumiracoxib $100 \mathrm{mg} /$ day versus celecoxib $200 \mathrm{mg} /$ day according to Patient Acceptable Symptom State (PASS). Pooled analysis of Lehmann et al. (2005) and Sheldon et al. (2005) (from Dougados et al. 2007)

\begin{tabular}{|c|c|c|c|c|}
\hline & $\begin{array}{l}\text { Lumiracoxib } 100 \mathrm{mg} / \text { day } \\
(\mathrm{n}=811)\end{array}$ & $\begin{array}{l}\text { Lumiracoxib } 100 \mathrm{mg} / \text { day with } \\
\text { initial dose of } 200 \mathrm{mg} / \text { day } \\
(\mathrm{n}=805)\end{array}$ & $\begin{array}{l}\text { Celecoxib } 200 \mathrm{mg} / \text { day } \\
(\mathrm{n}=813)\end{array}$ & $\begin{array}{l}\text { Placebo } \\
(n=806)\end{array}$ \\
\hline \multicolumn{5}{|c|}{ Pain intensity } \\
\hline Week 2 & $30.9^{a}$ & $33.5^{\mathrm{a}}$ & $29.8^{a}$ & 17 \\
\hline Week 13 & $43.3^{a}$ & $45.3^{\mathrm{a}}$ & $42.2^{\mathrm{b}}$ & 35.5 \\
\hline \multicolumn{5}{|c|}{ Disease activity } \\
\hline Week 2 & $29.6^{a}$ & $31.7^{\mathrm{a}}$ & $28.8^{\mathrm{a}}$ & 17.4 \\
\hline Week 13 & $42.8^{\mathrm{a}}$ & $43.9^{a}$ & $39.5^{\mathrm{a}}$ & 31.6 \\
\hline \multicolumn{5}{|c|}{ WOMAC function score } \\
\hline Week 2 & $32.3^{a}$ & $33.7^{\mathrm{a}}$ & $31.9^{a}$ & 19.1 \\
\hline Week 13 & $41.6^{\mathrm{a}}$ & $41.4^{\mathrm{a}}$ & $38.7^{\mathrm{a}}$ & 29.5 \\
\hline
\end{tabular}


A pooled analysis of the trials by Lehmann et al. (2005) and Sheldon et al. (2005) investigated the response to treatment using PASS (Dougados et al. 2007). PASS defines the symptom score beyond which patients consider themselves to be well $(0-100 \mathrm{~mm}$ VAS): $\leq 32.3 \mathrm{~mm}$ for pain intensity, $\leq 32 \mathrm{~mm}$ for disease activity, and $\leq 21.08$ for WOMAC function score. A significantly greater proportion of patients receiving lumiracoxib $100 \mathrm{mg} /$ day, with and without an initial dose of $200 \mathrm{mg} /$ day, were satisfied after 2 and 13 weeks compared with placebo; there was no significant difference between lumiracoxib and celecoxib 200 mg/day (Table 6). The response rate, measured by Minimally Clinical Important Improvement (MCII) in a patient's symptom score, was similar for lumiracoxib and celecoxib, and significantly greater than with placebo (Dougados et al. 2007).

\section{General tolerability}

The most common adverse events with lumiracoxib in clinical trials typically included headache and nasopharyngitis. As expected, Gl effects were also present, with nausea, diarrhea, dyspepsia, and upper abdominal pain being the most frequent minor Gl-related adverse events. The incidence of these adverse events was similar to that with celecoxib, diclofenac, ibuprofen, and naproxen. TARGET is the only long-term study assessing the safety of lumiracoxib over 52 weeks in a large population of patients (9117 receiving lumiracoxib treatment). Table 7 provides details of the adverse events experienced with lumiracoxib in this study. Discontinuation due to adverse events was similar between active treatment groups and placebo, although there were a greater number of discontinuations with diclofenac than lumiracoxib (14\% vs 3-5\%) in one RCT (Schnitzer et al. 2004a). In addition, Schnitzer et al. (2004a) reported that there was no dosage-related trend with lumiracoxib in the incidence of these adverse events.
Gl safety

Evidence suggests that lumiracoxib is associated with a comparable incidence of Gl complications to other COX-2 inhibitors, and a lower risk of upper Gl ulcer complications compared with ibuprofen or naproxen in patients not taking aspirin.

Level 1 evidence from a pooled analysis of 15 phase II and phase III RCTs in patients with OA or RA, demonstrated that treatment with lumiracoxib (100-1200 mg; 5439 patients) was associated with a significantly lower rate of perforations, obstructions, symptomatic ulcers, and bleedings than the nonselective NSAIDs (1.7 versus 13.7 events per 100 patient-years for lumiracoxib 200-400 mg) (Hawkey et al. 2006) (Table 8). The incidence was similar to that for celecoxib and rofecoxib (1.4 events per 100 patient-years).

Level 2 evidence from two RCTs of short duration (8 days) in healthy volunteers showed that lumiracoxib (200 or $800 \mathrm{mg}$ ) is associated with a lower incidence of gastric and duodenal erosion, compared with the nonselective NSAID naproxen (Rordorf et al. 2003; Atherton et al. 2004; Table 8). In addition, results from a study of 13 weeks' duration in patients with $O A$ demonstrated that lumiracoxib 200 or 400 mg was associated with a significant lower incidence of gastroduodenal ulcers ( $\geq 3 \mathrm{~mm}$ and $\geq 5 \mathrm{~mm}$ ) compared with the nonselective NSAID ibuprofen $(P<0.001$ and $P<0.005$, respectively), a similar incidence to that achieved with celecoxib (Hawkey et al. 2004a).

Gastric erosion is a surrogate marker for the development of peptic ulceration or other major Gl adverse effects. Therefore, the TARGET outcomes study in 18325 patients with OA was conducted to determine the clinical relevance of these

Table 7 | Level 2 evidence for the most common adverse events and discontinuations due to adverse events with lumiracoxib in patients with hip, knee or, hand OA (Schnitzer et al. 2004b)

\begin{tabular}{|c|c|c|c|c|}
\hline \multirow[t]{2}{*}{ Outcome } & \multicolumn{2}{|c|}{ Lumiracoxib vs ibuprofen substudy } & \multicolumn{2}{|c|}{ Lumiracoxib vs naproxen substudy } \\
\hline & $\begin{array}{l}\text { Lumiracoxib } \\
(n=4376)\end{array}$ & $\begin{array}{l}\text { Ibuprofen } \\
\text { (n=4397) }\end{array}$ & $\begin{array}{l}\text { Lumiracoxib } \\
(n=4741)\end{array}$ & $\begin{array}{l}\text { Naproxen } \\
(n=4730)\end{array}$ \\
\hline Total number of patients with AEs & $3586(82 \%)$ & $3559(81 \%)$ & $3647(77 \%)$ & $3717(79 \%)$ \\
\hline Total number of patients with SAEs & $297(7 \%)$ & $272(6 \%)$ & $291(6 \%)$ & $294(6 \%)$ \\
\hline Prespecified Gl events & $1855(42 \%)$ & $1851(42 \%)$ & $1785(38 \%)$ & $1988(42 \%)$ \\
\hline dyspepsia & $1230(28 \%)$ & $1205(27 \%)$ & $1037(22 \%)$ & $1119(24 \%)$ \\
\hline abdominal pain upper & $380(9 \%)$ & $452(10 \%)$ & $535(11 \%)$ & $695(15 \%)$ \\
\hline diarrhea & $285(7 \%)$ & $247(6 \%)$ & $273(6 \%)$ & $195(4 \%)$ \\
\hline nausea & $244(6 \%)$ & $261(6 \%)$ & $221(5 \%)$ & $264(6 \%)$ \\
\hline abdominal pain & $147(3 \%)$ & $153(4 \%)$ & $181(4 \%)$ & $187(4 \%)$ \\
\hline Discontinuations due to AEs (including SAEs) & $718(16 \%)$ & $802(18 \%)$ & $723(15 \%)$ & $855(18 \%)$ \\
\hline
\end{tabular}


Table 8 | Evidence for the incidence of GI erosions and ulcers with lumiracoxib

$\begin{array}{llll}\text { Reference } & \text { Design and population } & \text { Treatment (no.) Incidence of GI erosions and ulcers }\end{array}$

\section{Level 1 evidence}

Hawkey et al. 2006

Pooled analysis (15 phase II and III RCTs)

Pts with hip, knee, hand, or spine

OA, or symptomatic RA pts

1-52 wk, db, RCT

\section{Endoscopic studies: level 2 evidence}

Atherton et al. 2004 Healthy volunteers

$8 \mathrm{~d}, \mathrm{db}, \mathrm{dd}, \mathrm{RCT}$ (3-way

crossover; 18-d washout period)

$800 \mathrm{mg}$ LX od (24)

PL (24)

$500 \mathrm{mg}$ naproxen bid (24)

$\mathrm{PL}$ (20)

$500 \mathrm{mg}$ naproxen bid (20)

$200 \mathrm{mg} \mathrm{LX}$ od $(257)^{\mathrm{a}}$ $400 \mathrm{mg} L X$ od $(253)^{\mathrm{a}}$ $800 \mathrm{mg}$ ibuprofen tid $(248)^{\mathrm{a}}$

$200 \mathrm{mg} \mathrm{CX}$ od $(253)^{\mathrm{a}}$
Incidence of PUBs: 1.7 events per 100 patient-years, LX 200/400 mg; 1.4 events per 100 patient-years, celecoxib and rofecoxib; 13.7 events per 100 patient-years, nonselective NSAIDs

Frequency of symptomatic ulcers: $0.4 \%$ LX 200/400 mg, 0.4\% CX and $\mathrm{RX}, 2.5 \%$ nonselective NSAIDs

No subjects receiving $L X$ developed gastroduodenal erosions 3 subjects (12.5\%) on PL developed gastroduodenal erosions (1-6 erosions per individual) 18 subjects $(75 \%)$ on naproxen had a total of 255 gastroduodenal erosions (1-32 erosions per individual). Erosions were predominantly in the gastric antrum

No duodenal erosions (endoscopy grade 3 or above) with LX or PL $13 / 20(65 \%)$ pts had duodenal erosions with naproxen

No gastric erosions with LX

One subject in naproxen group developed an asymptomatic gastric ulcer One subject in PL group developed a grade 4 gastric erosion (6-10 erosions)

The cumulative incidence of gastroduodenal ulcers ( $\geq 3 \mathrm{~mm}$ diameter) was $4.3 \%$ and $4.0 \%$ for $200 \mathrm{mg} L X$ and $400 \mathrm{mg} \mathrm{LX}$, respectively (both $P<0.001$ vs ibuprofen), $15.7 \%$ for ibuprofen and $3.2 \%$ for $\mathrm{CX}$ The cumulative incidence of gastroduodenal ulcers ( $\geq 5 \mathrm{~mm}$ diameter) was $3.9 \%$ and $3.6 \%$ for $200 \mathrm{mg} \mathrm{LX}$ and $400 \mathrm{mg} L X$, respectively (both $P<0.005$ vs ibuprofen), $12.5 \%$ for ibuprofen and $2.8 \%$ for $\mathrm{CX}$ The number of pts with $>10$ gastroduodenal erosions was significantly greater with ibuprofen (6\%) compared with $200 \mathrm{mg} L X(1.2 \% ; P<0.01)$, $400 \mathrm{mg} L X(1.6 \%, P<0.05)$, and $\mathrm{CX}(2.4 \%, P<0.05)$

$72.4 \%$ and $76.7 \%$ of pts receiving $200 \mathrm{mg} L X$ and $400 \mathrm{mg} L X$, respectively had no visible injury of gastric mucosa compared with $55.2 \%$ of pts receiving ibuprofen (both $P<0.001$ vs ibuprofen) and $75.5 \%$ of pts receiving $\mathrm{CX}$ $83.7 \%$ and $87.4 \%$ of pts receiving $200 \mathrm{mg} \mathrm{LX}$ and $400 \mathrm{mg} L X$, respectively, had no visible injury of gastric mucosa compared with $74.2 \%$ of pts receiving ibuprofen $(P<0.05 \mathrm{LX} 200 \mathrm{mg}, P<0.001 \mathrm{LX} 400 \mathrm{mg}$ vs ibuprofen) and $87.7 \%$ of pts receiving $\mathrm{CX}$

Lower level mucosal injury: only one pt developed an esophageal ulcer (LX $400 \mathrm{mg}$ )

Number (incidence) of upper GI ulcer complications ${ }^{b}: 10(0.23 \%)$ LX vs 33 (0.75\%) ibuprofen (HR 0.29; $P=0.0006) ; 19(0.40 \%)$ LX vs $50(1.06 \%)$ naproxen (HR $0.37 ; P=0.0002)$. The majority of these $\mathrm{Gl}$ complications were presented as melena or hematochezia

Number (incidence) of upper Gl ulcer complications in nonaspirin population ${ }^{b}$ : $5 / 3401$ (0.15\%) LX vs 28/3431 (0.82\%) ibuprofen (HR 0.17; $P=0.0003$ ); $9 / 3549$ (0.25\%) LX vs 36/3537 (1.02\%) naproxen (HR 0.24; $P=0.0001$ )

Number (incidence) of upper GI ulcer complications in aspirin population ${ }^{\mathrm{b}}$ : $5 / 975$ (0.51\%) LX vs 5/966 (0.52\%) ibuprofen (HR 0.92; $P=0.9008)$; $10 / 1192(0.84 \%)$ LX vs $14 / 1193(1.17 \%)$ naproxen (HR $0.73 ; P=0.4502)$

Number (incidence) of symptomatic uncomplicated ulcers ${ }^{\mathrm{c}}: 58 / 9117$ (0.64\%) LX vs 103/9127 (1.13\%) nonselective NSAIDs (HR 0.55; $P=0.0003$ )

aModified safety population (defined as all patients who underwent at least one postbaseline endoscopy).

befinite or probable upper Gl ulcer complications (clinically significant bleeding, perforation, or obstruction from erosive or ulcer disease).

cUlcers discovered when endoscopy was done for dyspepsia.

bid, twice daily; CX, celecoxib; d, day; db, double-blind; dd, double-dummy; GI, gastrointestinal; HR, hazard ratio; LX, lumiraoxib; mc, multicenter; NSAIDs, nonsteroidal antiinflammatory

drugs; OA, osteoarthritis; od, once daily; PL, placebo; pts, patients; PUBs, perforations, obstructions, symptomatic ulcers, and bleedings; RA, rheumatoid arthritis; RCT, randomized controlled trial; RX, rofecoxib, RCT; tid, three times daily; wk, week. 
endoscopic findings (Schnitzer et al. 2004b; Hawkey et al. 2004b; Table 8). This study showed that patients receiving lumiracoxib at a dose of $400 \mathrm{mg}$, which is four-fold greater than the currently indicated dosage of $100 \mathrm{mg}$ for OA, had a significantly lower risk of upper Gl ulcer complications than patients receiving ibuprofen or naproxen. In contrast, in patients receiving low-dose aspirin (75-100 $\mathrm{mg} /$ day), the incidence of ulcer complications with lumiracoxib was similar to that with ibuprofen and naproxen, and provided no additional GI benefit.

It has been determined that using lumiracoxib rather than ibuprofen or naproxen for 1 year would prevent one severe upper Gl event for every 170 patients treated (nonaspirin and aspirin populations combined) (Bannwarth \& Berenbaum 2005). In comparison, in the nonaspirin population lumiracoxib would prevent one severe upper Gl event for every 139 patients treated.

\section{Cardiovascular safety}

Weak level 1 evidence from a meta analysis of 22 RCTs ranging from $\geq 1$ week and up to 1 year in duration in patients with either OA or RA found no evidence that lumiracoxib was associated with a significant increase in risk for a CV event compared with naproxen, other NSAIDs (diclofenac, ibuprofen, celecoxib, rofecoxib) or placebo (Matchaba et al. 2005; Table 9). A total of 15 studies were in patients with OA. The incidence of CV events in patients with OA is provided in Table 10.

\section{Table 9 | The risk of CV events with lumiracoxib in patients with OA or RA: results from a meta analysis of all RCTs $\geq 1$ week and up to 1 year (Matchaba et al. 2005)}

\begin{tabular}{|c|c|c|c|}
\hline CV event & $\begin{array}{l}\text { RR (lumiracoxib vs } \\
\text { placebo) }\end{array}$ & $\begin{array}{l}\text { RR (lumiracoxib } \\
\text { vs naproxen) }\end{array}$ & $\begin{array}{l}\text { RR (lumiracoxib } \\
\text { vs nonnaproxen } \\
\text { NSAID }\end{array}$ \\
\hline APTC & $\begin{array}{l}1.08 \\
(15 / 7011 \mathrm{LX} ; 6 / 3234 \\
\text { PL) }\end{array}$ & $\begin{array}{l}1.49 \\
(50 / 5964 \mathrm{LX} \\
31 / 5411 \\
\text { naproxen) }\end{array}$ & $\begin{array}{l}0.83 \\
(22 / 6126 \mathrm{LX} \\
24 / 5058 \\
\text { nonnaproxen) }\end{array}$ \\
\hline $\begin{array}{l}\text { Myocardial } \\
\text { event }\end{array}$ & $\begin{array}{l}1.27 \\
\text { (6/7011 LX; 2/3234 PL) }\end{array}$ & $\begin{array}{l}1.69 \\
(22 / 5964 \mathrm{LX} \\
12 / 5411 \\
\text { naproxen) }\end{array}$ & $\begin{array}{l}0.80 \\
(7 / 6126 \mathrm{LX} \\
8 / 5058 \\
\text { nonnaproxen) }\end{array}$ \\
\hline Stroke & $\begin{array}{l}0.59 \\
(4 / 7011 \text { LX; 3/3234 PL) }\end{array}$ & $\begin{array}{l}1.42 \\
(20 / 5964 \mathrm{LX} \\
13 / 5411 \\
\text { naproxen) }\end{array}$ & $\begin{array}{l}0.91 \\
(9 / 6126 \mathrm{LX} \\
9 / 5058 \\
\text { nonnaproxen) }\end{array}$ \\
\hline \multicolumn{4}{|c|}{$\begin{array}{l}\text { APTC, Antiplatelet Trialists' Collaboration composite CV endpoint of myocardial infarction, } \\
\text { stroke (ischemic and hemorrhagic), and CV death; CV, cardiovascular; LX, lumiracoxib; } \\
\text { NSAID, nonsteroidal antiinflammatory drug; PL, placebo; RCT, randomized controlled trial; } \\
\text { RR, relative risk. }\end{array}$} \\
\hline
\end{tabular}

The evidence from this meta analysis is weak because the majority of the studies reported were of 4 or 13 weeks' duration. Few CV events were reported with lumiracoxib during this period (Table 10), and it is unlikely that evidence of CV events would be demonstrated after such a short period of drug exposure. Only TARGET was of adequate duration (1 year) to provide meaningful level 2 evidence for the incidence of CV events with lumiracoxib. However, 1 year may be not sufficient to determine long-term CV risk, and studies of longer duration may be required. Furthermore, a major shortcoming of TARGET was the exclusion of patients with a history of CV disease. Only patients at increased risk of coronary heart disease were eligible provided that they had received lowdose aspirin (75-100 mg daily) for a minimum of 3 months prior to randomization.

Of the 34668 patients included in this meta analysis, 18325 were from TARGET. This RCT was divided into two substudies to determine the CV safety of lumiracoxib (400 mg once daily; $\mathrm{n}=4741$ ) compared with naproxen (500 mg twice daily; $\mathrm{n}=4730$ ) and lumiracoxib (400 mg once daily; $n=4376$ ) compared with ibuprofen (800 mg three times daily; $n=4397$ ) (Farkouh et al. 2004).

Approximately $24 \%(n=4326)$ of patients participating in TARGET were taking low-dose aspirin, although only $36 \%(n=1561)$ of these were classified as high CV risk. The incidence of MI did not differ significantly between lumiracoxib and either ibuprofen or naproxen, irrespective of aspirin use. However, in the naproxen substudy in patients not taking low-dose aspirin fewer patients had Mls in the naproxen 500 mg twice-daily group (four events, $0.11 \%$ ) compared with the lumiracoxib group (ten events, $0.28 \%$ ) $(P=0.1454)$. The dose of lumiracoxib used in TARGET was four times the current recommended dose of $100 \mathrm{mg}$ for OA, therefore, it may be expected that any $\mathrm{CV}$ risk would be reduced at the indicated dose.

In healthy subjects, lumiracoxib does not affect the ex-vivo antiplatelet aggregation activity of low-dose aspirin (Jermany et al. 2005). In contrast, naproxen inhibits platelet aggregation and has been suggested to have an antithrombotic effect, suppressing thromboxane production to a similar level as lowdose aspirin (Capone et al. 2004). This property may explain the reduced incidence of $\mathrm{MI}$ with naproxen compared with lumiracoxib, although this remains debatable. Farkouh et al. (2004) highlighted that the absence of a placebo arm in this study made it difficult to ascertain the real risk of $\mathrm{Ml}$ with lumiracoxib alone.

Level 1 evidence from another meta analysis revealed no significant difference in cerebrovascular event risk in patients taking lumiracoxib compared with placebo, nonselective NSAIDs, or other COX-2 inhibitors. A slightly higher risk was found compared specifically with naproxen (OR 1.09, 95\% Cl $0.58,2.04)$ and ibuprofen (OR 1.31, 95\% Cl 0.57, 2.98), although this did not reach significance (Chen \& Ashcroft 2006). The authors concluded that any increase in CV risk with COX-2 inhibitors must therefore be mainly due to risk of MI.

A further meta analysis that included six lumiracoxib trials revealed no effect on arrhythmia risk, although the authors stated that the results were inconclusive due to the limited number of studies (Zhang et al. 2006). 


\section{Table 10 | Incidence and relative risk of CV events with lumiracoxib in patients with OA: results from a meta analysis of RCTs} (Matchaba et al. 2005) ${ }^{\mathrm{a}}$

\begin{tabular}{|c|c|c|c|c|c|}
\hline Reference & $\begin{array}{l}\text { Duration of study } \\
\text { (wk) }\end{array}$ & Treatment (dose) & $\begin{array}{l}\text { Number of APTC events } \\
\text { (safety population) }\end{array}$ & $\begin{array}{l}\text { Number of myocardial } \\
\text { events (safety population) }\end{array}$ & $\begin{array}{l}\text { Number of stroke events } \\
\text { (safety population) }\end{array}$ \\
\hline Berenbaum et al. 2005 & 1 & $\begin{array}{l}\text { LX (200/400 mg) } \\
\text { PL }\end{array}$ & $\begin{array}{l}\text { 0/204 } \\
0 / 103 \\
\text { RR: no events }\end{array}$ & $\begin{array}{l}0 / 204 \\
0 / 103 \\
\text { RR: no events }\end{array}$ & $\begin{array}{l}\text { 0/204 } \\
0 / 103 \\
\text { RR: no events }\end{array}$ \\
\hline Wittenberg et al. 2003 & 1 & $\begin{array}{l}\mathrm{LX}(400 \mathrm{mg}) \\
\mathrm{PL}\end{array}$ & $\begin{array}{l}1 / 144 \\
0 / 75 \\
\text { RR not estimable }\end{array}$ & $\begin{array}{l}1 / 144 \\
0 / 75 \\
\text { RR not estimable }\end{array}$ & $\begin{array}{l}0 / 144 \\
0 / 75 \\
\text { RR: no events }\end{array}$ \\
\hline $\begin{array}{l}\text { Benevolenskaya } \\
\text { et al. } 2003\end{array}$ & 4 & $\begin{array}{l}\text { LX (100 mg) } \\
\text { PL }\end{array}$ & $\begin{array}{l}\text { 0/122 } \\
\text { 0/122 } \\
\text { RR: no events }\end{array}$ & $\begin{array}{l}0 / 122 \\
0 / 122 \\
\text { RR: no events }\end{array}$ & $\begin{array}{l}0 / 122 \\
0 / 122 \\
\text { RR: no events }\end{array}$ \\
\hline Grifka et al. 2004 & 4 & $\begin{array}{l}\text { LX (200/400 mg) } \\
\text { PL }\end{array}$ & $\begin{array}{l}\text { 0/398 } \\
\text { 0/196 } \\
\text { RR: no events }\end{array}$ & $\begin{array}{l}\text { 0/398 } \\
\text { 0/196 } \\
\text { RR: no events }\end{array}$ & $\begin{array}{l}\text { 0/398 } \\
\text { 0/196 } \\
\text { RR: no events }\end{array}$ \\
\hline Schnitzer et al. $2004 a$ & 4 & $\begin{array}{l}\text { LX (50/100/200/400 mg) } \\
\text { PL } \\
\text { Diclofenac }(75 \mathrm{mg})\end{array}$ & $\begin{array}{l}0 / 392 \\
0 / 97 \\
\text { 0/94 } \\
\text { RR: no events }\end{array}$ & $\begin{array}{l}0 / 392 \\
0 / 97 \\
\text { 0/94 } \\
\text { RR: no events }\end{array}$ & $\begin{array}{l}0 / 392 \\
0 / 97 \\
\text { 0/94 } \\
\text { RR: no events }\end{array}$ \\
\hline Hawkey et al. 2003 & 6 & $\begin{array}{l}\mathrm{LX}(400 \mathrm{mg}) \\
\mathrm{RX}(25 \mathrm{mg})\end{array}$ & NR & NR & NR \\
\hline Fleischmann et al. 2005 & 13 & $\begin{array}{l}\text { LX (200/400 mg) } \\
\text { PL } \\
\text { CX (200 mg) }\end{array}$ & $\begin{array}{l}2 / 925 \\
0 / 231 \\
\text { NR } \\
\text { RR not estimable }\end{array}$ & $\begin{array}{l}2 / 925 \\
0 / 231 \\
\text { NR } \\
\text { RR not estimable }\end{array}$ & $\begin{array}{l}0 / 925 \\
0 / 231 \\
\text { NR } \\
\text { RR: no events }\end{array}$ \\
\hline Tannenbaum et al. 2004 & 13 & $\begin{array}{l}\text { LX (200/400 mg) } \\
\text { PL }\end{array}$ & $\begin{array}{l}1 / 978 \\
0 / 243\end{array}$ & $\begin{array}{l}1 / 978 \\
0 / 243\end{array}$ & $\begin{array}{l}0 / 978 \\
0 / 243\end{array}$ \\
\hline $\begin{array}{l}\text { Novartis data on file } \\
2002^{b}\end{array}$ & 13 & $\begin{array}{l}\mathrm{LX}(400 \mathrm{mg}) \\
\mathrm{PL} \\
\mathrm{RX}(25 \mathrm{mg})\end{array}$ & $\begin{array}{l}1 / 205 \\
2 / 204 \\
\text { NR } \\
\text { RR } 0.46\end{array}$ & $\begin{array}{l}0 / 205 \\
1 / 204 \\
\text { NR } \\
\text { RR not estimable }\end{array}$ & $\begin{array}{l}0 / 205 \\
1 / 204 \\
\text { NR } \\
\text { RR not estimable }\end{array}$ \\
\hline Hawkey et al. 2004 & 13 & $\begin{array}{l}\text { LX }(200 / 400 \mathrm{mg}) \\
\text { Ibuprofen }(800 \mathrm{mg}) \\
\text { CX }(200 \mathrm{mg})\end{array}$ & $\begin{array}{l}1 / 524 \\
1 / 260 \\
\text { NR } \\
\text { RR } 0.46\end{array}$ & $\begin{array}{l}1 / 524 \\
1 / 260 \\
\text { NR } \\
\text { RR } 0.46\end{array}$ & $\begin{array}{l}0 / 524 \\
0 / 260 \\
\text { NR } \\
\text { RR: no events }\end{array}$ \\
\hline Sheldon et al. 2005 & 13 & $\begin{array}{l}\mathrm{LX}(100 \mathrm{mg}) \\
\mathrm{PL}\end{array}$ & $\begin{array}{l}1 / 776 \\
1 / 382 \\
\text { RR } 0.43\end{array}$ & $\begin{array}{l}0 / 776 \\
0 / 382 \\
\text { RR: no events }\end{array}$ & $\begin{array}{l}0 / 776 \\
1 / 382 \\
\text { RR not estimable }\end{array}$ \\
\hline Lehmann et al. 2005 & 13 & $\begin{array}{l}\text { LX }(100 \mathrm{mg}) \\
\text { PL2/840 } \\
1 / 424 \\
\operatorname{RR} 0.96\end{array}$ & $\begin{array}{l}2 / 840 \\
1 / 424 \\
\text { RR } 0.96\end{array}$ & $\begin{array}{l}0 / 840 \\
1 / 424 \\
\text { RR not estimable }\end{array}$ & $\begin{array}{l}1 / 840 \\
0 / 424 \\
\text { RR not estimable }\end{array}$ \\
\hline Schell et al. $2003^{c}$ & 39 & $\begin{array}{l}\text { LX (100 mg) } \\
\text { LX (400 mg) } \\
\text { CX (200 mg) }\end{array}$ & NR & NR & NR \\
\hline $\begin{array}{l}\text { Pavelka et al. } 2005 \\
\text { (abstract) }\end{array}$ & 39 & $\begin{array}{l}\text { LX (100 mg) } \\
\text { CX (200 mg) }\end{array}$ & NR & NR & NR \\
\hline Farkouh et al. $2004^{d}$ & 52 & $\begin{array}{l}\text { LX }(400 \text { mg) } \\
\text { Naproxen }(500 \text { mg) } \\
\text { LX (400 mg) } \\
\text { lbuprofen }(800 \text { mg) }\end{array}$ & $\begin{array}{l}40 / 4741 \\
27 / 4730 \\
\text { RR } 1.44 \\
19 / 4376 \\
23 / 4397 \\
\text { RR } 0.79\end{array}$ & $\begin{array}{l}18 / 4741 \\
10 / 4730 \\
\text { RR } 1.75 \\
5 / 4376 \\
7 / 4397 \\
\text { RR } 0.68\end{array}$ & $\begin{array}{l}16 / 4741 \\
12 / 4730 \\
\text { RR } 1.30 \\
8 / 4376 \\
9 / 4397 \\
\text { RR } 0.85\end{array}$ \\
\hline \multicolumn{6}{|c|}{$\begin{array}{l}\text { aThese data are not available in the primary references. Data for the incidence of CV events with the COX-2 comparators (celecoxib or rofecoxib) were not reported, therefore it is not provided } \\
\text { in this table. } \\
\text { 'Published as Berenbaum et al. } 2005 . \\
\text { 'Extension of Tannenbaum et al. } 2004 . \\
\text { 'TARGET study. } \\
\text { APTC, Antiplatelet Trialists' Collaboration composite CV endpoint of myocardial infarction, stroke (ischemic and hemorrhagic), and CV death; COX-2, cyclooxygenase-2; CV, cardiovascular; } \\
\text { CX, celecoxib; LX, lumiracoxib; NR, not reported; OA, osteoarthritis; PL, placebo; RR, relative risk; RCT, randomized controlled trial; RX, rofecoxib; TARGET, Therapeutic Arthritis Research and } \\
\text { Gastrointestinal Event Trial; wk, week. }\end{array}$} \\
\hline
\end{tabular}


Table 11 | Level 2 evidence for liver function outcomes with lumiracoxib in healthy volunteers and OA patients

\begin{tabular}{|c|c|c|c|}
\hline Reference & Design and population & Treatment (no.) & Liver function outcomes \\
\hline Rordorf et al. 2003 & $\begin{array}{l}\text { Healthy male volunteers } \\
8 \mathrm{~d}, \mathrm{db}, \mathrm{dd}, \mathrm{RCT}\end{array}$ & $\begin{array}{l}200 \text { mg LX bid (20) } \\
\text { PL (20) } \\
500 \text { mg naproxen bid (20) }\end{array}$ & $\begin{array}{l}\text { ALT/AST elevations (fold increase over ULN ranged } \\
\text { from } 1.1 \text { to } 4.2 \text { for AST and } 1.1 \text { to } 2.2 \text { for ALT): } 4 \text { pts LX, } \\
4 \text { pts PL, } 1 \text { pt naproxen }\end{array}$ \\
\hline Lehmann et al. 2005 & $\begin{array}{l}\text { Knee OA } \\
13 \text { wk, db, dd, mc, RCT }\end{array}$ & $\begin{array}{l}100 \mathrm{mg} \text { LX od }(420) \\
100 \mathrm{mg} \text { LX od }+200 \mathrm{mg} \\
\text { loading dose LX od for first } 2 \text { wk (420) } \\
200 \mathrm{mg} \text { CX od (420) } \\
\text { PL (424) }\end{array}$ & $\begin{array}{l}\text { Incidence of liver function parameter elevations }>3 \times \\
\text { ULN: } 0.2 \% 100 \mathrm{mg} \mathrm{LX}+\text { loading dose, } 0.5 \% \mathrm{PL}, 0.2 \% \mathrm{CX}\end{array}$ \\
\hline Sheldon et al. 2005 & $\begin{array}{l}\text { Knee OA } \\
13 \text { wk, db, dd, mc, RCT }\end{array}$ & $\begin{array}{l}100 \mathrm{mg} \text { LX od (391) } \\
100 \mathrm{mg} \text { LX od }+200 \mathrm{mg} \\
\text { loading dose LX od for first } 2 \text { wk (385) } \\
200 \mathrm{mg} \text { CX od (393) } \\
\text { PL (382) }\end{array}$ & $\begin{array}{l}\text { Incidence ALT/AST levels }>3 \times \text { ULN: } 0.3 \% \text { for both } \\
\text { LX and CX, 0\% PL }\end{array}$ \\
\hline $\begin{array}{l}\text { Tannenbaum et al. } \\
2004\end{array}$ & $\begin{array}{l}\text { Knee OA } \\
13 \text { wk, db, dd, mc, RCT }\end{array}$ & $\begin{array}{l}200 \mathrm{mg} \text { LX od (487) } \\
400 \mathrm{mg} \text { LX od }(491) \\
200 \mathrm{mg} \mathrm{CX} \text { od }(481) \\
\text { PL (243) }\end{array}$ & $\begin{array}{l}\text { Incidence ALT/AST levels >3 x ULN: } 2 \text { pts LX } \\
200 \text { mg, } 3 \text { pts LX } 400 \mathrm{mg}, 4 \text { pts CX. None were } \\
\text { accompanied by clinical symptoms } \\
\text { All cases resolved either while receiving study drug } \\
\text { or after treatment had stopped. No clinical symptoms } \\
\text { were associated with elevations }\end{array}$ \\
\hline $\begin{array}{l}\text { Fleischmann et al. } \\
2005\end{array}$ & $\begin{array}{l}\text { Knee OA } \\
13 \text { wk, db, dd, mc, RCT }\end{array}$ & $\begin{array}{l}200 \mathrm{mg} \text { LX od (462) } \\
400 \mathrm{mg} \text { LX od }(463) \\
200 \mathrm{mg} \mathrm{CX} \text { od }(444) \\
\text { PL (231) }\end{array}$ & $\begin{array}{l}\text { Incidence ALT >3 x ULN: } 0.4 \% 200 \mathrm{mg} \text { LX, } 2.6 \% \\
400 \text { mg LX, 0.0\% CX, 0.4\% PL } \\
\text { Incidence AST >3 x ULN: } 0.2 \% 200 \mathrm{mg} \text { LX, } 1.1 \% \\
400 \mathrm{mg} \text { LX, } 0.2 \% \mathrm{CX}, 0.4 \% \mathrm{PL} \\
\text { All cases resolved either while receiving study drug } \\
\text { or after treatment had stopped. No clinical symptoms } \\
\text { were associated with elevations }\end{array}$ \\
\hline Schnitzer et al. 2004b & $\begin{array}{l}\text { Hip, knee, hand, or spine OA } \\
52 \text { wk, db, dd, mc, RCT }\end{array}$ & $\begin{array}{l}400 \text { mg LX od (4376) } \\
800 \text { mg ibuprofen tid (4397) } \\
400 \text { mg LX od ( } 4741) \\
500 \text { mg naproxen bid (4730) }\end{array}$ & $\begin{array}{l}\text { Proportion of pts with ALT/AST concentrations }>3 \times \text { ULN was } \\
\text { greater with LX than with ibuprofen or naproxen }[2.57 \% \\
\text { ( } n=230) \text { vs } 0.63 \% \text { ( } n=56)(H R 3.97)] \\
9 \text { pts had probable or possible drug-induced clinical } \\
\text { hepatitis: } 6 \text { events }(0.07 \%) \text { LX, } 2 \text { events }(0.05 \%) \text { ibuprofen, } \\
1 \text { event }(0.02 \%) \text { naproxen } \\
\text { Abnormalities resolved on cessation of treatment. No cases of } \\
\text { liver failure, transplantation, or death attributable to drug- } \\
\text { induced hepatitis reported }\end{array}$ \\
\hline
\end{tabular}

ALT, alanine aminotransferase; AST, aspartate aminotransferase; bid, twice daily; CX, celecoxib; d, day; db, double-blind; dd, double-dummy; Gl, gastrointestinal; HR, hazard ratio;

LX, lumiracoxib; mc, multicenter; od, once daily; OA, osteoarthritis; PL, placebo; pts, patients; RCT, randomized controlled trial; tid, three times daily; ULN, upper limit of normal; wk, week.

\section{Liver function}

Level 2 evidence from six RCTs in healthy volunteers (one study) and patients with OA (five studies) showed that a small proportion of patients receiving a high dose of lumiracoxib $(400 \mathrm{mg})$ experienced clinically relevant elevations in the liver function enzymes alanine aminotransferase (ALT) and aspartate aminotransferase (AST), which resolved on cessation of treatment (Table 11). These elevations were greater than in patients receiving ibuprofen or naproxen (Schnitzer et al. 2004b). However, at the indicated dose of $100 \mathrm{mg}$, the incidence was similar to that of celecoxib and placebo.

There is some evidence that higher doses of lumiracoxib may be associated with changes in liver function in some patients. Eleven cases of severe hepatic adverse reactions, including nine cases of liver failure, two deaths, and three liver transplants, at least possibly related to lumiracoxib have been reported among the estimated 7 million patients who have been exposed to the drug since its launch
(MHRA 2007). Most of these reports were associated with doses of 200 to $400 \mathrm{mg} /$ day. Nevertheless, there has been one report of a transient liver reaction among the estimated 5400 patients receiving the drug at $100 \mathrm{mg}$ in the UK (MHRA 2007).

\section{Renal function}

The incidence of edema with lumiracoxib in five RCTs in patients with OA is shown in Table 12. Results from these studies showed that the incidence of edema with lumiracoxib did not appear to be dose-related and was infrequent in the majority of studies (ranging from $0 \%$ to $5 \%$ ), with an incidence similar to that with celecoxib, diclofenac, naproxen, and ibuprofen. The incidence was increased in one RCT (11.5\% for lumiracoxib $100 \mathrm{mg}$ ), but was also high for placebo $(4.1 \%)$ in this study.

Evidence from TARGET, in which $45 \%$ of patients had hypertension, also showed that lumiracoxib $400 \mathrm{mg}$ had a significantly less 
Table 12 | Level 2 evidence for the incidence of edema with lumiracoxib treatment in patients with OA

\begin{tabular}{|c|c|c|c|}
\hline Reference & Design & $\begin{array}{l}\text { Treatment } \\
\text { (no. patients) }\end{array}$ & $\begin{array}{l}\text { Number }(\%) \text { of } \\
\text { patients with } \\
\text { peripheral edema }\end{array}$ \\
\hline $\begin{array}{l}\text { Grifka } \\
\text { et al. } 2004\end{array}$ & $\begin{array}{l}\text { Hand OA } \\
4 \text { wk, db, mc, } \\
\text { RCT }\end{array}$ & $\begin{array}{l}200 \text { mg LX od (205) } \\
400 \text { mg LX od (193) } \\
\text { PL (196) }\end{array}$ & $\begin{array}{l}1(0.5) \\
0(0.0) \\
0(0.0)\end{array}$ \\
\hline $\begin{array}{l}\text { Schnitzer } \\
\text { et al. } 2004 a\end{array}$ & $\begin{array}{l}\text { Hip or knee OA } \\
\text { Phase II dose- } \\
\text { finding study } \\
4 \mathrm{wk}, \mathrm{db}, \mathrm{RCT}\end{array}$ & $\begin{array}{l}50 \text { mg LX bid (98) } \\
100 \text { mg LX bid (96) } \\
200 \text { mg LX bid (99) } \\
400 \text { mg LX od (99) } \\
75 \text { mg diclofenac } \\
\text { bid (94) } \\
\text { PL (97) }\end{array}$ & $\begin{array}{l}5(5.1) \\
11(11.5) \\
8(8.1) \\
8(8.1) \\
5(5.3) \\
4(4.1)\end{array}$ \\
\hline $\begin{array}{l}\text { Tannenbau } \\
\text { m et al. } \\
2004\end{array}$ & $\begin{array}{l}\text { Knee OA } \\
13 \text { wk, db, dd, } \\
\text { mc RCT }\end{array}$ & $\begin{array}{l}200 \text { mg LX od (487) } \\
400 \text { mg LX od (491) } \\
200 \text { mg CX od (481) } \\
\text { PL (243) }\end{array}$ & $\begin{array}{l}6(1.2) \\
4(0.8) \\
6(1.2) \\
4(1.6)\end{array}$ \\
\hline $\begin{array}{l}\text { Fleischman } \\
n \text { et al. } 2005\end{array}$ & $\begin{array}{l}\text { Knee OA } \\
13 \text { wk, db, dd, } \\
\text { mc RCT }\end{array}$ & $\begin{array}{l}200 \text { mg LX od (462) } \\
400 \text { mg LX od (463) } \\
200 \text { mg CX od (444) } \\
\text { PL (231) }\end{array}$ & $\begin{array}{l}11(2.4) \\
10(2.2) \\
12(2.7) \\
4(1.7)\end{array}$ \\
\hline $\begin{array}{l}\text { Zacher } \\
\text { et al. } 2005 \\
\text { (abstract) }\end{array}$ & $\begin{array}{l}\text { Hip, knee, hand, } \\
\text { or spine OA } \\
52 \text { wk, db, dd, } \\
\text { mc, RCT }\end{array}$ & $\begin{array}{l}400 \mathrm{mg} \text { LX od (4376) } \\
800 \mathrm{mg} \text { ibuprofen tid } \\
(4397) \\
400 \mathrm{mg} \text { LX od (4741) } \\
500 \mathrm{mg} \text { naproxen bid } \\
(4730)\end{array}$ & $\begin{array}{l}\text { Incidence in } \\
\text { nonaspirin } \\
\text { population: LX vs } \\
\text { ibuprofen/naproxen } \\
\text { (combined data) } \\
4.6 \% \text { vs } 4.7 \% \\
\text { Incidence in aspirin } \\
\text { population: LX vs } \\
\text { ibuprofen/naproxen } \\
\text { (combined data) } \\
5.0 \% \text { vs } 5.5 \%\end{array}$ \\
\hline
\end{tabular}

pronounced effect on systolic blood pressure (SBP) and diastolic blood pressure (DBP) compared with the nonselective NSAIDs (mean change from baseline SBP $+0.4 \mathrm{mmHg}$ vs $+2.1 \mathrm{mmHg}$; DBP $-0.1 \mathrm{mmHg}$ vs $+0.5 \mathrm{mmHg}$; both $P<0.0001$ ). Farkouh et al. (2004) suggested that this may be due to reduced sodium retention in patients receiving lumiracoxib.

Additional evidence from TARGET, published in abstract form, showed that the incidence of de-novo hypertension with lumiracoxib was low and similar to that with naproxen (Zacher et al. 2005; Table 13). The incidence of de-novo hypertension was greater with ibuprofen compared with lumiracoxib. Therefore, it is unlikely that the lower dose of lumiracoxib indicated for OA will increase hypertension. These effects were seen in patients who were also receiving concomitant aspirin.

A subsequent meta analysis that included six lumiracoxib trials in patients with OA or RA indicated no effect of the drug at a median dosage of $300 \mathrm{mg} /$ day on overall renal function (RR 1.07, 95\%
Table 13 | The incidence of de-novo hypertension with lumiracoxib treatment in patients with $\mathrm{OA}$ (level 2 evidence from TARGET; Zacher et al. 2005)

\begin{tabular}{|ll|}
\hline Population & $\begin{array}{l}\text { Incidence of de-novo } \\
\text { hypertension (\%) }\end{array}$ \\
\hline $\begin{array}{l}\text { Nonaspirin population } \\
\text { Lumiracoxib vs ibuprofen/naproxen } \\
\text { (combined data) }\end{array}$ & 5.0 vs $6.5(P<0.01)$ \\
Lumiracoxib vs ibuprofen \\
Lumiracoxib vs naproxen & 5.0 vs $7.9(P<0.01)$ \\
Aspirin population & 5.0 vs 5.2 \\
$\begin{array}{l}\text { Lumiracoxib vs ibuprofen/naproxen } \\
\text { (combined data) }\end{array}$ & 5.8 vs $6.5(P=0.593)$ \\
Lumiracoxib vs ibuprofen & 5.0 vs $7.2(P=0.267)$ \\
Lumiracoxib vs naproxen & 6.4 vs 6.1 \\
\hline OA, osteoarthritis & \\
\hline
\end{tabular}

Cl $0.68,1.70$ ), including peripheral edema (RR $1.31,95 \% \mathrm{Cl} 0.75$, 2.31), hypertension ( $\mathrm{RR} 0.94,95 \% \mathrm{Cl} 0.42,2.12$ ), or renal dysfunction demonstrated by serum urea, creatinine, kidney disease or renal failure (RR $0.75,95 \%$ Cl 0.17, 3.32) (Zhang et al. 2006).

\section{Drug interactions}

Lumiracoxib is metabolized by the cytochrome P450 isoform 2C9 (CYP 2C9) (Mangold et al. 2004; Rordorf et al. 2005). However, lumiracoxib has no significant pharmacokinetic interactions with the CYP 2C9 substrate, warfarin (Bonner et al. 2003), or the CYP 2C9 inhibitor, fluconazole (Scott et al. 2004a). Therefore, it is anticipated that lumiracoxib has a low potential for drug-drug interactions with other CYP 2C9 substrates. Coadministration of lumiracoxib with common treatments for dyspepsia (an aluminum hydroxide/magnesium hydroxide antacid or omeprazole) has also been demonstrated to have no clinically significant effect on the single-dose plasma pharmacokinetics of lumiracoxib (Scott et al. 2004b). In addition, moderate hepatic impairment and mild to moderate renal impairment do not appear to influence lumiracoxib exposure (Kalbag et al. 2004; Rordorf et al. 2005).

\section{Economic evidence and resource utilization}

There is no published evidence of the cost effectiveness of lumiracoxib.

The acquisition cost of lumiracoxib in the UK is less than that of celecoxib (net price $100 \mathrm{mg} \times 30$ lumiracoxib £17.24 vs £21.55 for celecoxib), and greater than that of nonselective NSAIDs, many of which are in generic form (BNF 2005).

Looking beyond acquisition cost, reducing the incidence (and subsequent management) of clinically significant ulcer complications should have a beneficial effect on overall costs of 
managing OA. A cost-effectiveness and cost-utility analysis in patients at average risk of $\mathrm{Gl}$ and $\mathrm{CV}$ events showed that an NSAID in combination with a PPI costs an incremental \$US45 350 per additional ulcer complication avoided and \$US309 666 per quality-adjusted life year (QALY) gained compared with an NSAID alone (Spiegel et al. 2005). A costutility analysis for the long-term treatment of OA or RA has suggested that the use of the first-generation COX-2 inhibitors rofecoxib or celecoxib is only cost effective in patients with a previous bleeding ulcer compared with the nonselective NSAID naproxen (Spiegel et al. 2003). Cost comparisons of lumiracoxib with nonselective NSAIDs (with or without gastroprotective agents) in both patients at low and high risk of Gl complications are needed to determine whether the degree of risk reduction offsets their increased cost.

Clinical evidence demonstrates that lumiracoxib provided no additional Gl benefit compared with ibuprofen and naproxen in patients receiving low-dose aspirin (75-100 mg/day) (Schnitzer et al. 2004b). Therefore, lumiracoxib is not expected to be cost effective in patients taking aspirin.

As detailed in the Current therapy options section, nonselective NSAIDs and the COX-2 inhibitors rofecoxib and celecoxib have been shown to increase BP. In patients with OA or RA, a 3 $\mathrm{mmHg}$ increase in SBP over 1 year was associated with a $4 \%$ increase in the incidence of ischemic heart disease and stroke events (Singh et al. 2003). A study estimating the clinical and economic impact of a $2.26 \%$ increase in SBP in 7.3 million American adults with $\mathrm{OA}$ and treated hypertension showed that maintaining BP control would avoid $>30000$ deaths from stroke and 25000 coronary deaths. This would theoretically result in >449 000 person-years of life saved and \$US1.4 billion in direct healthcare cost savings (Grover et al. 2005). Limited clinical evidence suggests that lumiracoxib does not increase the incidence of de-novo hypertension, and that lumiracoxib did not cause increased BP compared with patients receiving nonselective NSAIDs. The cost benefit of this potential reduction in CV events would be of interest. A clinical trial investigating the effect of lumiracoxib $100 \mathrm{mg}$ once daily compared with ibuprofen $600 \mathrm{mg}$ three times daily on ambulatory BP in patients with $\mathrm{OA}$ and controlled hypertension is currently underway (http://www.clinicaltrials.gov).

\section{Patient group/population}

Level 1 evidence from a Cochrane review of acetaminophen in OA concluded that the relative superiority of nonselective NSAIDs over acetaminophen is most marked in patients with moderate to severe levels of pain (Towheed et al. 2006). As acetaminophen is inexpensive and well tolerated it is likely that it will continue to be used as an initial therapy option for the initial treatment of patients with mild to moderate OA pain. In patients who fail to achieve adequate symptomatic relief with acetaminophen, nonselective NSAIDs are currently the preferred option, with COX-2 inhibitors used as an alternative, particularly in patients at high risk of developing serious Gl adverse effects.

\section{Patients with CV risk factors}

It is currently recommended that all NSAIDs, including COX-2 inhibitors, are used with caution in patients with risk factors for CV disease such as hypertension, hyperlipidemia, diabetes, and smoking. However, a large proportion of patients with $\mathrm{OA}$ are likely to be elderly, and have CV risk factors or established CV disease.

The Third National Health And Nutrition Examination Survey (NHANES III) reported that over $47 \%$ of US adults with OA were older than 65 years of age and had a high prevalence of CV risk factors (Singh et al. 2002). Approximately $40 \%$ of individuals aged $\geq 35$ years with OA had hypertension, $20 \%$ smoked, $11 \%$ had diabetes, and $32 \%$ had hypercholesterolemia. Therefore, a need remains for a COX-2 inhibitor that can be used safely in this highrisk population.

Data from TARGET demonstrate that lumiracoxib did not increase the incidence of MI, stroke, or other thrombotic CV events in patients that were treated for up to 1 year. This trial included patients with CV risk factors; $45 \%$ were hypertensive, $8 \%$ had diabetes, and $20 \%$ had dyslipidemia. Therefore, lumiracoxib may be used with caution in patients with these comorbidities (EMEA 2005b). However, patients with established CV disease were excluded from TARGET.

Low-dose aspirin has a cardioprotective (antiplatelet) effect due to inhibition of the COX-1 enzyme. Therefore, it is prescribed to many patients with CV disease. Data from TARGET show that in patients receiving low-dose aspirin, lumiracoxib provided no benefit in ulcer complication reduction over ibuprofen or naproxen. Topol and Falk (2004) suggest that it is hard to justify the use of COX-2 inhibitors in these patients. In addition, NICE guidelines suggest that prescribing COX-2 inhibitors in combination with aspirin is not justified as they would not provide a GI benefit (NICE 2001).

\section{Patients with hepatic impairment}

Patients treated with lumiracoxib $400 \mathrm{mg}$ experienced reversible elevations in liver function parameters. It is therefore likely that there is a low potential for lumiracoxib at the indicated dosage of $100 \mathrm{mg}$ to increase the incidence of liver dysfunction in the outpatient setting. Liver function monitoring is advised before starting and every month during lumiracoxib treatment (MHRA 2007). The drug is contraindicated in patients with, or at high risk of, hepatic impairment (MHRA 2007).

\section{Patients with renal impairment}

NHANES III estimated that $37 \%$ of patients with OA have renal impairment, but fewer than $1 \%$ experience renal failure. Clinical evidence has shown that lumiracoxib is associated with a low incidence of peripheral edema. The summary of product characteristics states that under conditions of compromised renal perfusion, administration of lumiracoxib may impair renal function (EMEA 2005b). Therefore, it is recommended that monitoring of renal function in these patients is considered. 


\section{Dosage, administration, and formulations}

In the EU, lumiracoxib is indicated for symptomatic relief of OA at a recommended dose of $100 \mathrm{mg}$ once daily. It is advised that the patients' need for symptomatic relief and response to therapy is reevaluated periodically. The summary of product characteristics advises that lumiracoxib is used with caution in patients most at risk of developing a Gl complication with NSAIDs, and in patients with a history of cardiac failure, left ventricular dysfunction, hypertension, or preexisting edema. It warns that patients with significant CV risk factors should only be treated with lumiracoxib after careful consideration. Lumiracoxib is contraindicated in patients with current hepatic disease, previous drug-induced elevations of alanine aminotransferase (ALT)/aspartate aminotransferase (AST), ALT/AST $>1.5 \times$ upper limit of normal (ULN) before treatment or $>3 \times$ ULN during treatment, or taking other drugs that may impair hepatic function (MHRA 2007).

\section{Clinical value}

Clear evidence indicates that lumiracoxib is as effective as the nonselective NSAIDs diclofenac, naproxen, and ibuprofen, and the COX-2 inhibitor celecoxib for the management of pain in patients with $\mathrm{OA}$, as shown by improvements in pain intensity and joint stiffness. Evidence suggests that lumiracoxib improves quality of life, as shown by improvements in WOMAC scores for the ability to perform daily activities and improvements in SF-36 scores for physical functioning and mental health. However, the economic impact of lumiracoxib on the treatment of $O A$, as compared to other currently available medications, has not been reported.

There is a need to identify the OA patient population most likely to benefit from COX-2 inhibitors as an alternative to nonselective NSAIDs. Evidence indicates that lumiracoxib is associated with a lower risk of upper Gl complications, which is maintained for up to 1 year's treatment, compared with ibuprofen or naproxen. Patients already receiving aspirin would not have a GI benefit from treatment with lumiracoxib because of the inhibitory effects of aspirin on gastric COX-1. It is expected that lumiracoxib will be included in updated guidelines as an alternative therapy to nonselective NSAIDs in combination with PPIs, and as an alternative primary therapy to celecoxib in patients at high-risk of developing serious Gl adverse effects. The impact of lumiracoxib on hospitalization and death rates resulting from Gl complications compared to other NSAIDs has not been reported. Given the higher acquisition costs of lumiracoxib compared with nonselective NSAIDs, many of which are available generically, these data are important to evaluate any potential cost benefits.

There is moderate evidence that a high dose of lumiracoxib (400 mg) has a similar risk of CV events as ibuprofen and naproxen over a 1-year period. Further evidence will confirm if the CV safety is sustained in the longer term with approved doses (100 mg). The impact of lumiracoxib on CV events relative to other COX-2 inhibitors has not been reported. More evidence is required to determine whether lumiracoxib has an advantage over other COX-2 inhibitors with regard to $\mathrm{CV}$ risk profile. In addition, the impact of lumiracoxib on CV events in patients with $\mathrm{CV}$ disease has not been reported. As the majority of patients with $\mathrm{OA}$ are aged $\geq 65$ years, often have CV risk factors, and receive aspirin medication, more evidence evaluating the expected role of lumiracoxib in the management of these patients is needed.

The potential for adverse hepatic effects with lumiracoxib limit its usefulness. The drug was withdrawn from Australia in August 2007 following reports of serious adverse liver reactions (Therapeutic Goods Administration 2007), although this was at doses greater than the $100 \mathrm{mg}$ approved in the EU. However, two cases of adverse liver reactions at this dose resulted in the drug being withdrawn in Canada in October 2007 (Health Canada 2007), and the drug has not been approved by the FDA because of concerns over its potential to impair liver function (Anon. 2007). In the EU, the drug is contraindicated in patients with current, or at risk from, hepatic dysfunction (MHRA 2007). The risk of adverse liver reactions appears to be small, with 11 case reports of severe reactions in the estimated 7 million patients who have taken the drug since it was introduced (MHRA 2007). Furthermore, the safety data reviewed by the FDA revealed that $0.85 \%$ of patients receiving lumiracoxib 100 mg for 12 months had ALT and AST elevations $>3 \times$ ULN, a rate considered to be similar to that seen with other NSAIDs (Anon. 2007).

In summary, lumiracoxib is a new COX-2 inhibitor providing an alternative treatment option for the management of OA, with comparable efficacy and $\mathrm{CV}$ safety to nonselective NSAIDs, but with a lower risk of Gl complications in patients not taking aspirin. It may provide an acceptable risk-benefit balance in patients with a higher incidence of GI complications, including those with ulcers or being treated with anticoagulants.

\section{Acknowledgments}

The authors declare that they have no conflict of interest.

\section{References}

ACR (American College of Rheumatism). Recommendations for the medical management of osteoarthritis of the hip and knee. Arthritis Rheum. 2000;43:1905-1915.

Aldington S, Shirtcliffe P, Weatherall M, Beasley R. Systematic review and meta-analysis of the risk of major cardiovascular events with etoricoxib therapy. NZ Med J. 2005;118:U1684.

Anon. FDA rejects Novartis' COX-2 inhibitor Prexige. Available at: http://www.fdanews.com/newsletter/article?issueld=10818\&articleld=99378 (accessed October 9, 2007).

Atherton C, Jones J, McKaig B, et al. Pharmacology and gastrointestinal safety of lumiracoxib, a novel cyclooxygenase-2 selective inhibitor: an integrated study. Clin Gastroenterol Hepatol. 2004;2:113-120.

Aw T-J, Joseph S, Liew D, Krum H. Meta-analysis of cyclooxygenase-2 inhibitors and their effects on blood pressure. Arch Intern Med. 2005;165:490-496.

Badley EM. The economic burden of musculoskeletal disorders in Canada is similar to that for cancer, and may be higher. J Rheumatol. 1995;22:204-206. 
Bannwarth B, Berenbaum F. Clinical pharmacology of lumiracoxib, a secondgeneration cyclooxygenase 2 selective inhibitor. Expert Opin Investig Drugs. 2005;14:521-533.

Benevolenskaya L, Tuzan S, Hagin E, et al. Lumiracoxib is effective in relieving symptoms of knee or hip osteoarthritis after 4 weeks of treatment: results from a randomized, placebo-controlled trial. Ann Rheum Dis. 2003;62(Suppl. 1):270. Abstract FRI0246.

Berenbaum F, Grifka J, Brown JP, et al. Efficacy of lumiracoxib in osteoarthritis: a review of nine studies. $J$ Int Med Res. 2005;33:21-41.

Bombardier C, Laine L, Reicin A, et al. Comparison of upper gastrointestinal toxicity of rofecoxib and naproxen in patients with rheumatoid arthritis. N Engl J Med. 2000;343:1520-1528.

Bonner J, Branson J, Milosavljev S, Rordorf C, Scott G. Co-administration of lumiracoxib and warfarin does not alter the pharmacokinetic profile of $R$ - or S-warfarin. Ann Rheum Dis. 2003;62(Suppl. 1):264. Abstract FRI0225.

Bresalier RS, Sandler RS, Quan H, et al. Cardiovascular events associated with rofecoxib in a colorectal adenoma chemoprevention trial. The Adenomatous Polyp Prevention on Vioxx (APPROVe) trial investigators. $\underline{N \text { Engl }}$ J Med. 2005;352:1092-1102.

BNF (British National Formulary). BNF 50. London: BMJ Publishing Group Ltd; September 2005. Available at: http://bnf.org (accessed January 26, 2006).

Capone ML, Tacconelli S, Sciulli MG, et al. Clinical pharmacology of platelet, monocyte, and vascular cyclooxygenase inhibition by naproxen and low-dose aspirin in healthy subjects. Circulation. 2004;109:1468-1471.

Chen L-C, Ashcroft DM. Do selective COX-2 inhibitors increase the risk of cerebrovascualr events? A meta analysis of randomized controlled trials. J Clin Pharm Ther. 2006;31:565-576.

Dougados M, Leclaire P, van der Heijde D, Bloch DA, Bellamy N, Altman RD. Response criteria for clinical trials on osteoarthritis of the knee and hip: a report of the Osteoarthritis Research Society International Standing Committee for Clinical Trials response criteria initiative. Osteoarthritis Cartilage. 2000;8:395-403.

Dougados M, Moore A, Yu S, Gitton X. Evaluation of the patient acceptable symptom state in a pooled analysis of two multicentre, randomized,

double-blind, placebo-controlled studies evaluating lumiracoxib and celecoxib in patients with osteoarthritis. Arthritis Res Ther. 2007;9:RII.

Dubois RN, Abramson SB, Crofford L, et al. Cyclooxygenase in biology and disease. FASEB J. 1998;12:1063-1073.

EMEA (European Medicines Agency). European Medicines Agency concludes action on COX-2 inhibitors. London: European Medicines Agency press release; June 2005a. Available at:

http://www.emea.eu.int/pdfs/human/press/pr/20776605en.pdf (accessed January 26, 2006).

EMEA (European Medicines Agency). Lumiracoxib Summary of Product Characteristics. Surrey: Novartis Pharmaceuticals UK Ltd; 2005b. Available at: http://www.emea.eu.int/pdfs/human/epar/Lumiracoxib.pdf (accessed February 13, 2006).

Farkouh ME, Kirshner H, Harrington RA, et al. Comparison of lumiracoxib with naproxen and ibuprofen in the Therapeutic Arthritis Research and Gastrointestinal Event Trial (TARGET), cardiovascular outcomes: randomised controlled trial. Lancet. 2004;364:675-684.

FDA (Food and Drug Administration). FDA panel finds no safety benefit for Celebrex. Scrip World Pharm News. 2001;(2616):19.

FDA (Food and Drug Administration). February 16-18, 2005 Joint Meeting with the Arthritis Drug Advisory Committee. Rockville, MD: Food and Drug Administration; 2005a. Available at:

http://www.fda.gov/ohrms/dockets/ac/cder05.html\#DrugSafetyRiskMgmt (accessed January 25, 2006).

FDA (Food and Drug Administration). FDA Public Health Advisory. FDA announces important changes and additional warnings for COX-2 selective and non-selective non-steroidal anti-inflammatory drugs (NSAIDs). Rockville, MD: Food and Drug Administration; 2005b. Available at:

http://www.fda.gov/cder/drug/advisory/COX2.htm (accessed January 25, 2006).

Fitzgerald GA, Patrono C. The coxibs, selective inhibitors of cyclooxygenase2. N Engl J Med. 2001;345:433-442.
Fleischmann R, Sheldon E, Maldonado-Cocco J, Dutta D, Yu S, Sloan VS. Lumiracoxib is effective in the treatment of osteoarthritis of the knee: a prospective randomized 13-week study versus placebo and celecoxib. Clin Rheumatol. 2005;25:42-53.

Forman JP, Stampfer MJ, Curhan GC. Non-narcotic analgesic dose and risk of incident hypertension in US women. Hypertension. 2005;46:500-507.

Gambaro G, Perazella MA. Adverse renal effects of anti-inflammatory agents: evaluation of selective and nonselective cyclooxygenase inhibitors. J Intern Med. 2003;253:643-652.

Grifka JK, Zacher J, Brown JP, et al. Efficacy and tolerability of lumiracoxib versus placebo in patients with osteoarthritis of the hand. Clin Exp Rheumatol. 2004;22:589-596.

Grifka J, Sinclair N, Brenol JC, Hoexter G, Matchaba P, Sloan VS. Therapeutic arthritis research and gastrointestinal event trial (TARGET) of lumiracoxib vs NSAIDS: efficacy in different joints. Ann Rheum Dis. 2005;64(Suppl. iii):480.

Grover SA, Coupal L, Zowall H. Treating osteoarthritis with cyclooxygenase2 -specific inhibitors. What are the benefits of avoiding blood pressure destabilization? Hypertension. 2005;45:92-97.

Gupta S, Hawker GA, Laporte A, Croxford R, Coyte PC. The economic burden of disabling hip and knee osteoarthritis $(\mathrm{OA})$ from the perspective of individuals living with this condition. Rheumatology. 2005;44:1531-1537.

Hawkey C, Hoexter G, Richard D, et al. Lumiracoxib, a novel cyclooxygenase-2 selective inhibitor, has improved gastrointestinal safety and tolerability compared with nonselective, nonsteroidal antiinflammatory drugs: a pooled analysis. Arthritis Rheum. 2003;48(Suppl. 9):79. Abstract 87.

Hawkey CC, Svoboda P, Fiedorowicz-Fabrycy IF, et al. Gastroduodenal safety and tolerability of lumiracoxib compared with ibuprofen and celecoxib in patients with osteoarthritis. J Rheumatol. 2004a;31:1804-1810.

Hawkey CJ, Farkouh M, Gitton X, Ehrsam E, Huels J, Richardson P. Therapeutic arthritis research and gastrointestinal event trial of lumiracoxib study design and patient demographics. Aliment Pharmacol Ther. 2004b;20:51-63.

Hawkey CJ, Gitton X, Hoexter G, Richard D, Weinstein W. Gastroenterology tolerability of lumiracoxib in patients with osteoarthritis and rheumatoid arthritis. Clin Gastroenterol Hepatol. 2006;4:57-66.

Health Canada. Withdrawal of market authorization for Prexige. Available at: http://www.hc-sc.gc.ca/ahc-asc/media/advisories-

avis/2007/2007_141_e.html (accessed October 9, 2007).

Hippisley-Cox J, Coupland C. Risk of myocardial infarction in patients taking cyclo-oxygenase-2 inhibitors or conventional non-steroidal anti-inflammatory drugs: population based nested case-control analysis. BMJ. 2005;330:1366-1372.

Hippisley-Cox J, Coupland C, Logan R. Risk of adverse gastrointestinal outcomes in patients taking cyclo-oxygenase-2 inhibitors or conventional non-steroidal anti-inflammatory drugs: population based nested case-control analysis. BMJ. 2005;331:1310-1316.

Huang JQ, Sridhar S, Hunt RH. Role of Helicobacter pylori infection and non-steroidal anti-inflammatory drugs in peptic-ulcer disease: a metaanalysis. Lancet. 2002;359:14-22.

Hudson M, Richard H, Pilote L. Differences in outcomes of patients with congestive heart failure prescribed celecoxib, rofecoxib, or non-steroidal anti-inflammatory drugs: population based study. BMJ. 2005;330:1370-1376.

Jermany J, Branson J, Schmouder R, Guillaume M, Rordorf C. Lumiracoxib does not affect the ex vivo antiplatelet aggregation activity of low-dose aspirin in healthy subjects. J Clin Pharmacol. 2005;45:1172-1178.

Johnson AG, Nguyen TV, Day RO. Do nonsteroidal anti-inflammatory drugs affect blood pressure? A meta-analysis. Ann Intern Med. 1994;121:289-300.

Jones SC. Relative thromboembolic risks associated with COX-2 inhibitors. Ann Pharmacother. 2005;39:1249-1259.

Kalbag J, Yeh C-M, Milosavljev S, Lasseter K, Oberstein S, Rordorf C. No influence of moderate hepatic impairment on the pharmacokinetics of lumiracoxib, an oral COX-2 selective inhibitor. Pharmacol Res. 2004;50:181-186. 
Kurth T, Hennekens $\mathrm{CH}$, Sturmer T, et al. Analgesic use and risk of subsequent hypertension in apparently healthy men. Arch Intern Med. 2005;165:1903-1909. Laine L. Approaches to nonsteroidal anti-inflammatory drug use in the high-risk patient. Gastroenterology. 2001;120:594-606.

Lazzaroni M, Porro GB. Gastrointestinal side-effects of traditional non-steroidal anti-inflammatory drugs and new formulations. Aliment Pharmacol Ther. 2004;20(Suppl. 2):48-58.

Leardini G, Salaffi F, Caporali R, Canesi B, Rovati L, Montanelli R; Italian Group for Study of the Costs of Arthritis. Direct and indirect costs of osteoarthritis of the knee. Clin Exp Rheumatol. 2004;22:699-706.

Lehmann R, Brzosko M, Kopsa P, et al. Efficacy and tolerability of lumiracoxib $100 \mathrm{mg}$ once daily in knee osteoarthritis: a 13-week, randomized, double-blind study vs. placebo and celecoxib. Curr Med Res Opin. 2005;21:517-526.

Mangold JB, Gu H, Rodriguez LC, Bonner J, Dickson J, Rordorf C.

Pharmacokinetics and metabolism of lumiracoxib in healthy male subjects. Drug Metab Disposition. 2004;32:566-571.

Matchaba P, Gitton X, Krammer G, et al. Cardiovascular safety of lumiracoxib: a meta-analysis of all randomized controlled trials $\geq 1$ week and up to 1 year in duration of patients with osteoarthritis and rheumatoid arthritis. Clin Ther. 2005;27:1196-1214.

MHRA (Medicines and Healthcare Products Regulatory Agency). New (interim) restrictions on prescription of lumiracoxib, following concerns over liver safety. Available at:

http://www.mhra.gov.uk/home/idcplg?ldcService=SS_GET_PAGE\&useSecondar $y=$ true\&ssDocName $=$ CON2032098\&ssTargetNodeld=221 (accessed October 9, 2007).

Mitchell JA, Warner TD. Cyclo-oxygenase-2: pharmacology, physiology, biochemistry and relevance to NSAID therapy. Br J Pharmacol.

\section{9;128:1121-1132.}

NICE (National Institute for Health and Clinical Excellence). Guidance on the use of cyclo-oxygenase (Cox) II selective inhibitors, celecoxib, rofecoxib, meloxicam and etodolac for osteoarthritis and rheumatoid arthritis.

London: National Institute for Health and Clinical Excellence; July 2001.

Available at: http://www.nice.org.uk/pdf/coxiifullguidance.pdf (accessed December 2, 2005).

Pavelka K, Zamani O, Alten R, Yu S, Litschig S, Sloan VS. Lumiracoxib is effective and well tolerated in the long-term treatment of knee osteoarthritis. Ann Rheum Dis. 2005;64(Suppl. III):353. Abstract FRI0319.

Pendleton A, Arden N, Dougados M, et al. EULAR recommendations for the management of knee osteoarthritis: report of a task force of the Standing Committee for International Clinical Studies Including Therapeutic trials (ESCISIT). Ann Rheum Dis. 2000;59:936-944.

Pham T, van der Heijde D, Lassere M, et al. Outcome variables for osteoarthritis clinical trials: The OMERACT-OARSI set of responder criteria. $J$ Rheumatol. 2003;30:1648-1654.

Pope JE, Anderson JJ, Felson DT. A meta-analysis of the effects of nonsteroidal anti-inflammatory drugs on blood pressure. Arch Intern Med. 1993;153:477-484.

Reginster J-Y. The prevalence and burden of arthritis. Rheumatology. 2002;41(Suppl. 1):3-6.

Rordorf C, Kellett N, Mair S, et al. Gastroduodenal tolerability of lumiracoxib vs placebo and naproxen: a pilot endoscopic study in healthy male subjects. Aliment Pharmacol Ther. 2003;18:533-541.

Rordorf CM, Choi L, Marshall P, Mangold JB. Clinical pharmacology of lumiracoxib: a selective cyclo-oxygenase-2 inhibitor. Clin Pharmacokinet. 2005;44:1247-1266.

Scheiman JM, Fendrick AM. Practical approaches to minimizing gastrointestinal and cardiovascular safety concerns with COX-2 inhibitors and NSAIDS. Arthritis Res Ther. 2005;7(Suppl. 4):S23-S29.

Schell E, Boucher L, Tamasi L, et al. Long-term efficacy and tolerability of lumiracoxib in osteoarthritis of the knee. Ann Rheum Dis. 2003;62(Suppl. 1):264. Abstract FRI0224.

Schnitzer TJ, Beier J, Geusens P, et al. Efficacy and safety of four doses of lumiracoxib versus diclofenac in patients with knee or hip primary osteoarthritis: a phase II, four-week, multicenter, randomized, double-blind, placebocontrolled trial. Arthritis Rheum. 2004a;51:549-557.
Schnitzer TJ, Burmester GR, Mysler E, et al. Comparison of lumiracoxib with naproxen and ibuprofen in the Therapeutic Arthritis Research and Gastrointestinal Event Trial (TARGET), reduction in ulcer complications: randomized controlled trial. Lancet. 2004b;364:665-674.

Scott G, Yih L, Yeh CM, Milosavljev S, Laurent A, Rordorf C. Lumiracoxib: pharmacokinetic and pharmacodynamic profile when coadministered with fluconazole in healthy subjects. J Clin Pharmacol. 2004a;44:193-199.

Scott G, Vinluan Reynolds C, Milosavljev S, Langholff W, Shenouda M, Rordorf C. Lack of effect of omeprazole or of an aluminium hydroxide/magnesium hydroxide antacid on the pharmacokinetics of lumiracoxib. Clin Pharmacokinet. 2004b;43:341-348.

Sheldon E, Beaulieu A, Paster Z, Dutta D, Yu S, Sloan VS. Efficacy and tolerability of lumiracoxib in the treatment of osteoarthritis of the knee: a 13-week, randomized, double-blind comparison with celecoxib and placebo. Clin Ther. 2005;27:64-77.

Silverstein FE, Faich G, Goldstein JL, et al. Gastrointestinal toxicity with celecoxib vs nonsteroidal anti-inflammatory drugs for osteoarthritis and rheumatoid arthritis. A randomized controlled trial. Celecoxib Long-term Arthritis Safety Study. JAMA. 2000;284:1247-1255.

Singh G, Ramey DR, Terry R, Triadafilopoulos G. NSAID-related effects on gastrointestinal tract: an ever widening spectrum. Arthritis Rheum. 1997;40:S93-S98.

Singh G, Miller JD, Lee FH, Pettitt D, Russell MW. Prevalence of cardiovascular disease risk factors among US adults with self-reported osteoarthritis: data from the Third National Health and Nutrition Examination Survey. Am J Manag Care. 2002;8:S383-S391.

Singh G, Miller JD, Huse DM, Pettitt D, D’Agostino RB, Russell MW. Consequences of increased systolic blood pressure in patients with osteoarthritis and rheumatoid arthritis. J Rheumatol. 2003;30:714-719. Singh G, Triadafilopoulos G. Appropriate choice of proton pump inhibitor therapy in the prevention and management of NSAID-related gastrointestinal damage. Int J Clin Pract. 2005;59:1210-1217.

Solomon SD, McMurray JJ, Pfeffer MA, et al. Cardiovascular risk associated with celecoxib in a clinical trial for colorectal adenoma prevention. $\mathrm{NEngl} \mathrm{J}$ Med. 2005;352:1133-1135.

Spiegel BMR, Targownik L, Dulai GS, Gralnek IM. The cost-effectiveness of cyclooxygenase-2 selective inhibitors in the management of chronic arthritis. Ann Intern Med. 2003;138:795-806.

Spiegel BM, Chiou CF, Ofman JJ. Minimizing complications from nonsteroidal anti-inflammatory drugs: cost-effectiveness of competing strategies in varying risk groups. Arthritis Rheum. 2005;53:185-197.

Tannenbaum H, Berenbaum F, Reginster JY, et al. Lumiracoxib is effective in the treatment of osteoarthritis of the knee: a 13 week, randomised, double blind study versus placebo and celecoxib. Ann Rheum Dis. 2004;63:1419-1426.

Therapeutic Goods Administration. Medicines regulator cancels registration of anti inflammatory drug, lumiracoxib (Prexige). Available at: http://www.hcsc.gc.ca/ahc-asc/media/advisories-avis/2007/2007_141_e.html (accessed October 9, 2007).

Topol EJ, Falk GW. A coxib a day won't keep the doctor away. Lancet. 2004;364:639-640.

Towheed TE, Maxwell L, Judd MG, Catton M, Hochberg MC, Wells G. Acetaminophen for osteoarthritis. Cochrane Database Syst Rev. 2006;(1):CD004257.pub 2. DOI:10.1002/14651858.CD004257.pub2.

Vergara M, Catalan M, Gisbert JP, Calvet X. Meta-analysis: role of Helicobacter pylori eradication in the prevention of peptic ulcer in NSAID users. Pharmacol Ther. 2005;21:1411-1418.

Ware JE. SF-36 health survey: manual and interpretation guide. Boston, MA: The Health Institute, New England Medical Center; 1993.

Warner TD, Mitchell JA. Cyclooxygenases: new forms, new inhibitors, and lessons from the clinic. FASEB J. 2004;18:790-804.

Whelton A, Fort JG, Puma JA, Normandin D, Bello AE, Verburg KM; SUCCESS VI Study Group. Cyclooxygenase-2-specific inhibitors and cardiorenal function: a randomized, controlled trial of celecoxib, and rofecoxib in older hypertensive osteoarthritis patients. Am J Ther. 2001;8:85-95. 


\section{Lumiracoxib | clinical impact review}

Whelton A, White WB, Bello AE, Puma JA, Fort JG; SUCCESS-VII Investigators. Effects of celecoxib and rofecoxib on blood pressure and edema in patients > or $=65$ years of age with systemic hypertension and osteoarthritis. $\underline{\mathrm{Am} \mathrm{J}}$ Cardiol. 2002;90:959-963.

Wittenberg RH, Schell E, Krehan G, et al. Prospective, randomized study on the first-dose analgesic effect of lumiracoxib in osteoarthritis of the knee. Ann Rheum Dis. 2003;62(Suppl. 1):265. Abstract FRI0229.

Wittenberg RH, Schell E, Krehan G, et al. First-dose analgesic effect of the cyclo-oxygenase-2 selective inhibitor lumiracoxib in osteoarthritis of the knee: a randomized, double-blind, placebo-controlled comparison with celecoxib [NCT00267215]. Arthritis Res Ther. 2006;8:R35.

Wolfe MM, Lichtenstein DR, Singh G. Gastrointestinal toxicity of nonsteroidal antiinflammatory drugs. N Engl J Med. 1999;340:1888-1899.

WHO (World Health Organization). The burden of musculoskeletal conditions at the start of the new millennium. Report of a WHO scientific group. Geneva: WHO Technical Report Series, 919; 2003, pp. 218. Available at: http://www.emro.who.int/ncd/publications/musculoskeletalconditions.pdf (accessed January 9, 2006).

Woolf AD, Pfleger B. Burden of major musculoskeletal conditions. Bull World Health Organ. 2003;81:646-656.

Zacher J, Hasler P, Mola EM, Mellein B, Krammer G, Gitton X. Therapeutic arthritis research and gastrointestinal event trial (TARGET) of lumiracoxib vs NSAIDS: incidence of de-novo hypertension and oedema. Ann Rheum Dis. 2005;64(Suppl. iii):483.

Zhang J, Ding EL, Song Y. Adverse effects of cyclooxygenase 2 inhibitors on renal and arrhythmia events. JAMA. 2006;296:1619-1632.

Correspondence: Paul Chrisp, Core Medical Publishing, Mere House, Brook Street, Knutsford, Cheshire WA16 8GP, UK or at editor@coreevidence.com 